Check for updates

Cite this: RSC Adv., 2019, 9, 33497

Received 9th August 2019

Accepted 24th September 2019

DOI: $10.1039 / c 9 r a 06210 f$

rsc.li/rsc-advances

\section{Asymmetric amination of $\alpha, \alpha$-dialkyl substituted aldehydes catalyzed by a simple chiral primary amino acid and its application to the preparation of a S1P $_{1}$ agonist $\uparrow$}

\author{
Qiong Xiao, (D) ${ }^{a}$ Yifan Tang, ${ }^{b}$ Ping Xie ${ }^{\star a}$ and Dali Yin*a \\ The chiral catalytic amination of an $\alpha, \alpha$-dialkyl substituted aldehyde usually proceeds with low \\ enantioselectivity. We selected naphthyl-L-alanine as the catalyst and observed improved \\ enantioselectivity for the amination. Using this method, racemic $\alpha$-methyl- $\alpha$-benzyloxypropanal was \\ aminated to give chiral serine derivatives in $74 \%$ ee, which was further increased to $>99 \%$ ee after \\ recrystallization. Moreover, we also successfully synthesized a chiral phosphonium salt 9 for the \\ preparation of one $\alpha$-substituted alaninol compound 14 as an S1P $_{1}$ agonist in high overall yield.
}

\section{Introduction}

$\alpha, \alpha$-Disubstituted amino alcohols, aldehydes and acids are important chiral building blocks in organic synthesis. They are routinely found in a number of peptides, ${ }^{1-5}$ natural products ${ }^{6,7}$ and pharmaceuticals. ${ }^{\mathbf{8} 9}$ Due to this importance, their synthesis has attracted sustained interest from the synthetic community. Existing methods for the asymmetric approach to scaffolds include classical Seebach's method, ${ }^{\mathbf{1 0 , 1 1}}$ auxiliary Strecker synthesis, ${ }^{12}$ and a variety of asymmetric phase transfer catalysis reactions. ${ }^{13}$

Recently, several methods have been reported describing the asymmetric Michael $\alpha$-amination of achiral aldehydes via proline catalysis, resulting in the products being obtained in good yields and excellent enantioselectivities. ${ }^{\mathbf{1 4 - 1 7}}$ However, these proline catalysts do not imbue high enantioselectivities in the amination of branched aldehydes. Wang et al. reported that 3-(1-naphthyl)-L-alanine (1d) successfully promoted the enantioselective $\alpha$-amination of branched aldehydes with azadicarboxylates to give $\alpha$-alkyl- $\alpha$-aryl disubstituted aldehydes in up to $99 \%$ ee. ${ }^{18}$ However, low enantioselectivities only $4-28 \%$ ee were obtained with $\alpha$-alkyl- $\alpha$-alkyl disubstituted, potentially owing to poor stereo-differentiation between the two $\alpha$-substituents. ${ }^{14}$ To some extent, the application of this kind of reaction is limited. In 2005, Barbas et al. reported higher

${ }^{a}$ State Key Laboratory of Bioactive Substance and Function of Natural Medicines, Beijing Key Laboratory of Active Substance Discovery and Druggability Evaluation, Department of Medicinal Chemistry, Institute of Materia Medica, Peking Union Medical College, Chinese Academy of Medical Sciences, Beijing 100050, PR China. E-mail: yindali@imm.ac.cn

${ }^{b}$ Beijing Union Pharmaceutical Factory, PR China

$\dagger$ Electronic supplementary information (ESI) available. See DOI: 10.1039/c9ra06210f stereoselectivities were possible utilizing proline derived tetrazole catalyst (1) for the amination of $\alpha$-alkyl- $\alpha$-benzyl disubstituted aldehydes. ${ }^{19}$ In addition, no further progress about the asymmetric amination of $\alpha$-alkyl- $\alpha$-alkyl disubstituted aldehydes had been reported.

\section{Results and discussion}

Herein, we report the asymmetric Machel $\alpha$-amination of $\alpha$-methyl$\alpha$-protected hydroxymethyl aldehydes and their subsequent reduction and cyclisation to afford oxazolidinones in good ee. We initially chose 3-(benzyloxy)-2-methylpropanal and dibenzyl azodicarboxylate (DBAD) as a model substrate to determine to optimal reaction conditions. When L-proline (1a) (30 mol\%) was used, ${ }^{14}$ the reaction was complete in 48 hours at room temperature and provided the amino aldehyde in 56\% yield, however we obtained poor enantioselectivities (32\% ee). To improve the enantioselectivity, we screened a number of catalysts (Fig. 1). For example, tetrazole catalyst (1b) (15 mol\%) in $\mathrm{CH}_{3} \mathrm{CN}$ provided $42 \%$ ee with $68 \%$ yield (Table 1 , entry 2). ${ }^{17}$ 3-(1-Naphthyl)-L-alanine catalyst (1d) $(15 \mathrm{~mol} \%)$ in $\mathrm{CH}_{3} \mathrm{CN}$ gave the amino aldehyde in $70 \%$ yield with $46 \%$ ee (Table 1 , entry 4$){ }^{18}$

We then turned our attention to the effects of solvents on both yield and enantioselectivities (Table 2). Among them, dioxane, MeOH, MTBE and THF (entries 9, 10, 7 and 8) were all tolerated and produce the desired oxazolidinones in moderate

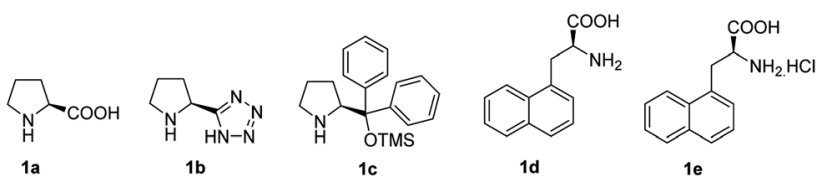

Fig. 1 Chiral catalysts. 
Table 1 Screening of chiral catalysts ${ }^{a}$ opposite enantiomer. yields. or reaction time.

Table 2 Screening of solvents ${ }^{a}$

\begin{tabular}{lllll}
\hline Entry & Catalyst & Time $(\mathrm{h})$ & Yield $^{b}(\%)$ & ee $^{c}(\%)$ \\
\hline 1 & 1a & 48 & 56 & 32 \\
2 & 1b & 12 & 68 & 42 \\
3 & 1c & 24 & 45 & $34^{d}$ \\
4 & 1d & 24 & 70 & 46 \\
5 & 1e & 24 & 53 & 44
\end{tabular}

${ }^{a}$ All reactions were carried out with aldehyde $(0.75 \mathrm{mmol})$, DBAD $(0.5$ $\mathrm{mmol})$, catalyst $(15 \mathrm{~mol} \%)$ in THF solvent $(4 \mathrm{~mL})$ at $\mathrm{rt}$ under argon, subsequent reduction and cyclisation to the oxazolidinone. ${ }^{b}$ Isolated yield. ${ }^{c}$ Determined by HPLC with a Chiralpak-OD column. ${ }^{d}$ With the

to good enantioselectivities. Of particular note, THF delivered the highest enantioselectivity (69\% ee) in synthetically useful

Furthermore, when lowering temperature to $0{ }^{\circ} \mathrm{C}$, we observed no improvement in enantioselectivity, however the reaction became notably more sluggish. Increasing catalyst loading up to $30 \mathrm{~mol} \%$ did not improve either enantioselectivity

With these optimized conditions in hand, we probed the substrate scope of the reaction (Table 3). In general, various oxazolidinones 5 were obtained in moderate to good yields (54$89 \%)$ and enantioselectivities $(24-73 \%$ ee $)$. The reactions showed poor enantioselectivities for $\alpha$-methyl- $\alpha$-ethyl and $\alpha$ methyl- $\alpha$-carbethoxy disubstituted aldehydes, but not for $\alpha$ -

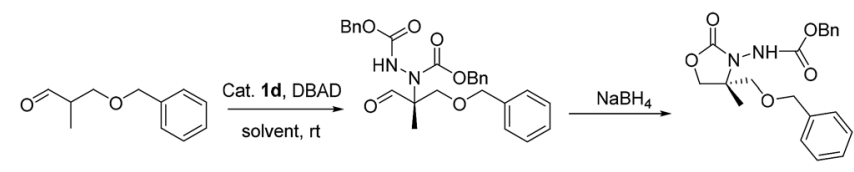

\begin{tabular}{lllll}
\hline Entry & Solvent & Time & Yield $^{b}(\%)$ & $\mathrm{ee}^{c}(\%)$ \\
\hline 1 & $n$-Hexane & 24 & 52 & 48 \\
2 & Toluene & 72 & 49 & 45 \\
3 & $\mathrm{CH}_{2} \mathrm{Cl}_{2}$ & 72 & 41 & 30 \\
4 & EtOAc & 24 & 67 & 54 \\
5 & $\mathrm{CH}_{3} \mathrm{OCH}_{2} \mathrm{CH}_{2} \mathrm{OCH}_{3}$ & 36 & 47 & 40 \\
7 & MTBE & 36 & 84 & 49 \\
8 & THF & 36 & 81 & 69 \\
9 & Dioxane & 36 & 76 & 57 \\
10 & MeOH & 24 & 69 & 57 \\
11 & Ethylene glycol & 24 & 42 & 57
\end{tabular}

${ }^{a}$ Reaction conditions: the azodicarboxylate (1 equiv.) was added to the aldehyde (1.5 equiv.), with catalyst ( $15 \mathrm{~mol} \%)$ in THF at $\mathrm{rt}$ for the stated period of time under argon. Reaction without isolation of intermediate.

${ }^{b}$ Isolated yield. ${ }^{c}$ Determined by chiral HPLC. methyl- $\alpha$-protected hydroxymethyl substituted aldehydes with aromatic ring. The results also showed that electronwithdrawing groups were more successful than electrondonating groups. Moreover, $p-\mathrm{F}$ and $p-\mathrm{CF}_{3}$ substituents both showed similar enantioselectivities. We then investigated differing azodicarboxylates and observed that di-p-chlorobenzyl azodicarboxylate (DCAD) provided the desired products in excellent yields $(90 \%)$ and good enantioselectivities (up to ee $74 \%$ ) while lower enantioselectivities were obtained with diethyl azadicarboxylate (DEAD) or diisopropyl azadicarboxylate (DIAD). We also observed good enantioselective control with catalysts bearing naphthalene rings. This may be due to the $\pi-\pi$ interaction between the aromatic ring of substrate and naphthalene ring limiting the conformation of the intermediate, thus improving the level of stereo-differentiation between the two $\alpha$-substituents. Additionally, when the azo reagent contained an aromatic ring this $\pi-\pi$ interaction may be further enhanced, resulting in the observed improvement of stereoselectivity.

Upon recrystallization from $90 \%$ ethanol, the aldehyde 4a-pClBn was obtained in $97 \%$ ee ( $60 \%$ yield) and $\mathbf{4 h}$ - $\boldsymbol{p}$-ClBn was obtained in $97 \%$ ee ( $65 \%$ yield), which was subsequently converted to oxazolidinone $\mathbf{5 a - p}$-ClBn in $>\mathbf{9 9 \%}$ ee and $\mathbf{5} \mathbf{h}$ - $\boldsymbol{p}$-ClBn was obtained in $98 \%$ ee respectively. The absolute configuration of 5- $R$ was determined to be $(R)$ on CD spectrum. Under ambient pressure, hydrogenation using $10 \% \mathrm{Pd} / \mathrm{C}$ in methanol/acetic acid, the benzyloxycarbonyl group was removed. Cleavage of the hydrazine moiety, 7 was accomplished by treating with $\mathrm{NaNO}_{2}$ (ref. 14) (Scheme 1). Alcohol 7 was treated with $p$-TsCl in pyridine, and the resulting tosylate was successively converted to iodide 8 with $\mathrm{NaI}$ in acetone under a reflux condition. ${ }^{20} 8$ with triphenylphosphine in DMF provided the desired phosphonium salt 9 in moderate yield as a stable white solid. ${ }^{20}$

Then we applied the chiral phosphonium salt 9 to the synthesis of biological active compound as $\mathrm{S}_{1} \mathrm{P}_{1}$ agonist $\mathbf{1 4}$. These types of compounds possessing a chiral 2-methyl-2aminoethanol have shown promise in recent years as the immunosuppressant. ${ }^{21,22}$ This compound is an analogue of SYL930, an immunosuppressant we have been reported before. ${ }^{23}$ SYL930 is currently in phase I clinical stage. The synthesis of $\mathbf{1 4}$ started from the aldehyde $\mathbf{1 1}$ in only a three step manipulation. ${ }^{24}$ Aldehyde $\mathbf{1 1}$ was synthesized in good yield from 4-bromobenzaldehyde and dinary pinacol borate ester 10 via Suzuki reaction with Pd-dimer (dibromobis(tri-tert-butylphosphine)dipalladium) as the catalyst. ${ }^{24}$ The Wittig reaction of 9 with 11 in dry THF at $-78^{\circ} \mathrm{C}$ for $3 \mathrm{~h}$ furnished the alkenes 12 in good yield. Subsequently reducing with $10 \% \mathrm{Pd} / \mathrm{C}$ in $\mathrm{MeOH}$ for $1 \mathrm{~h}$ afforded compound $\mathbf{1 3}$ in virtually quantitative yield after a flash-filtration. Finally, hydrolysis of the oxazolidinone part and then acidification with $1 \mathrm{M} \mathrm{HCl}$ in $\mathrm{Et}_{2} \mathrm{O}$ produced the chiral $\alpha$-substituted alaninol compound 14.

\section{Conclusions}

In this study, we presented an efficient asymmetric amination of branched racemic aldehydes catalyzed by the commercially available amino acid (3-(1-naphthyl)-L-alanine). Under the 
<smiles>[R]C(C)C=O</smiles>

2<smiles>[R20]OC(=O)/N=N/C([R20])=O</smiles><smiles>[R20]C(=O)NN(C(=O)O)C([R7])([R])C=O</smiles><smiles>[R20]C(=O)NN1C(=O)OCC1(C)C</smiles>

\begin{tabular}{|c|c|c|c|c|c|c|c|}
\hline Entry & $\mathrm{R}_{1}$ & $\mathrm{R}_{2}$ & Product & Time (h) & Yield $^{b}(\%)$ & $\mathrm{ee}^{c}(\%)$ & $\mathrm{ee}^{e}(\%)$ \\
\hline 1 & $\mathrm{BnOCH}_{2}$ & Et & 5a-Et & 38 & 79 & 57 & - \\
\hline 2 & $\mathrm{BnOCH}_{2}$ & $\mathrm{Bn}$ & $5 a-B n$ & 36 & 81 & 69 & - \\
\hline 4 & $\mathrm{BnOCH}_{2}$ & $p$-ClBn & $5 \mathrm{a}-p-\mathrm{ClBn}$ & 1 & 94 & 72 & $>99 \%$ \\
\hline 5 & $p-\mathrm{CH}_{3} \mathrm{BnOCH}_{2}$ & $\mathrm{Bn}$ & $5 b-B n$ & 38 & 54 & 48 & - \\
\hline 6 & $p-\mathrm{CH}_{3} \mathrm{BnOCH}_{2}$ & $p$-ClBn & $5 b-p-C l B n$ & 36 & 56 & 54 & - \\
\hline 9 & $p$-FBnOCH 2 & Et & 5d-Et & 37 & 75 & 56 & - \\
\hline 10 & $p$-FBnOCH ${ }_{2}$ & $\mathrm{Bn}$ & 5d-Bn & 36 & 80 & 68 & - \\
\hline 11 & $p$-FBnOCH ${ }_{2}$ & $p$-ClBn & 5d-p-ClBn & 48 & 89 & 70 & - \\
\hline 12 & $p-\mathrm{ClBnOCH}_{2}$ & Et & 5e-Et & 39 & 70 & 57 & - \\
\hline 13 & $p$-ClBnOCH ${ }_{2}$ & $\mathrm{Bn}$ & $5 e-B n$ & 36 & 63 & 59 & - \\
\hline 14 & $p-\mathrm{BrBnOCH}_{2}$ & $\mathrm{Bn}$ & $5 f-B n$ & 38 & 79 & 59 & - \\
\hline 19 & $p-\mathrm{CF}_{3} \mathrm{BnOCH}_{2}$ & Et & 5h-Et & 39 & 76 & 56 & - \\
\hline 20 & $p-\mathrm{CF}_{3} \mathrm{BnOCH}_{2}$ & $\mathrm{Bn}$ & $5 h-B n$ & 36 & 89 & 67 & - \\
\hline 21 & $p-\mathrm{CF}_{3} \mathrm{BnOCH}_{2}$ & $p$-ClBn & $4 h-p-C l B n$ & 48 & 90 & $74^{d}$ & $97 \%$ \\
\hline 22 & $p-\mathrm{CF}_{3} \mathrm{BnOCH}_{2}$ & $p$-ClBn & $5 h-p$-ClBn & 1 & 95 & 73 & $98 \%$ \\
\hline 23 & $p-\mathrm{NO}_{2} \mathrm{BnOCH}_{2}$ & $\mathrm{Bn}$ & $5 \mathrm{i}-\mathrm{Bn}$ & 48 & 77 & 55 & \\
\hline 24 & $\mathrm{THPOCH}_{2}$ & $\mathrm{Bn}$ & $5 \mathrm{j}-\mathrm{Bn}$ & 48 & 70 & 57 & \\
\hline 25 & $\mathrm{TrtOCH}_{2}$ & $\mathrm{Bn}$ & $5 \mathrm{k}-\mathrm{Bn}$ & 48 & - & - & \\
\hline 26 & Et & $\mathrm{Bn}$ & 5l-Bn & 48 & 78 & 37 & \\
\hline 27 & $\mathrm{CO}_{2} \mathrm{Et}$ & $\mathrm{Bn}$ & $4 m-B n$ & 48 & 76 & 24 & \\
\hline
\end{tabular}

${ }^{a}$ Reaction conditions: the azodicarboxylate (1 equiv.) was added to the aldehyde (1.5 equiv.), with catalyst (15 mol\%) in THF at rt for the stated period of time under argon. Reaction performed without isolating the intermediate. ${ }^{b}$ Isolated yield. ${ }^{c}$ Isolated by silica gel column chromatography. ${ }^{d}$ Determined by chiral HPLC. ${ }^{e}$ ee determined by chiral HPLC after recrystallization. Absolute configuration of $5-\boldsymbol{R}$ to determined be $(R)$ on CD spectrum.

optimized conditions, we obtained $\alpha$-methyl- $\alpha$-protected hydroxymethyl substituted aldehydes in high ee. Importantly, we developed an efficient catalytic method for synthesizing the Wittig reagent involving a chiral 2-methyl-2-aminoethanol structure that could be applied to other syntheses. Further, a new $\mathrm{S}_{1} \mathrm{P}_{1}$ agonist 14 has been obtained by this method in high overall yield.

\section{Experimental}

\section{General procedure for the synthesis of 4,4-disubstituted 3- alkoxycarbonylamino-oxazolidin-2-ones $(5-R)$ by one pot method}

Catalyst 1d (15 mol\% in respect to the azodicarboxylate) was added to a suspension of aldehydes $(2,1.5$ eq. in respect to the azodicarboxylate) and azodicarboxylate (3) in THF. The mixture stirred at rt under argon until the colour of the azodicarboxylate had disappeared. $\mathrm{NaBH}_{4}$ (3 eq. in respect to the azodicarboxylate) was added in portions at room temperature. The reaction mixture was stirred for $1 \mathrm{~h}$, and then it was quenched by adding $1 \mathrm{M} \mathrm{HCl}$ aq. until the mixture reached $\mathrm{pH} 7$, and it was extracted with $\mathrm{CH}_{2} \mathrm{Cl}_{2}$. The combined organic phases were dried over $\mathrm{Na}_{2} \mathrm{SO}_{4}$, and the solvent was evaporated under reduced pressure. The resulting crude was purified by flash chromatography on silica gel eluted with light petroleum ether-ethyl acetate mixture $(4: 1 \mathrm{v} / \mathrm{v})$ to afford products $\mathbf{5}-\boldsymbol{R}$ as oil or solid.

\section{3-Ethyloxycarbonylamino-4-methyl-4-benzyloxy-oxazolidin-2-one (5a-Et)}

White solid, yield 79\%; mp 50-55 ${ }^{\circ} \mathrm{C} ;{ }^{1} \mathrm{H}$ NMR (400 $\mathrm{MHz}, \mathrm{CDCl}_{3}$ ) $\delta 1.24\left(\mathrm{~m}, 6 \mathrm{H}, 2 \mathrm{CH}_{3}\right), 3.27$ (d, $\left.1 \mathrm{H}, J=8.0 \mathrm{~Hz}, \mathrm{CH}\right), 3.47$ (d, $1 \mathrm{H}, J=$ $12.0 \mathrm{~Hz}, \mathrm{CH}), 4.05$ (d, $1 \mathrm{H}, J=12.0 \mathrm{~Hz}, \mathrm{CH}), 4.14(\mathrm{q}, 2 \mathrm{H}, J=4.0 \mathrm{~Hz}$, $\mathrm{CH}_{2}$ ), 4.33 (d, $\left.1 \mathrm{H}, J=8.0 \mathrm{~Hz}, \mathrm{CH}\right), 4.51\left(\mathrm{~s}, 2 \mathrm{H}, \mathrm{CH}_{2}\right), 6.33$ (bs, $1 \mathrm{H}$, $\mathrm{NH}), 7.27-7.38\left(\mathrm{~m}, 5 \mathrm{H}, \mathrm{H}_{\mathrm{ar}}\right) ;{ }^{13} \mathrm{C} \mathrm{NMR}\left(100 \mathrm{MHz}, \mathrm{CDCl}_{3}\right) \delta 14.3,19.8$, 

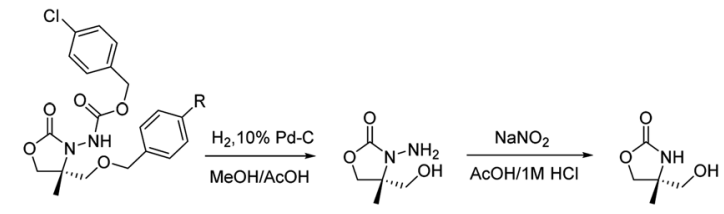

$$
\mathrm{R}=\mathrm{H},(R)-5 \mathrm{a}-\mathrm{p}-\mathrm{CIBn}
$$$$
\mathrm{R}=\mathrm{CF}_{3},(R)-5 \mathrm{~h}-\mathrm{p}-\mathrm{CIBn}
$$

1.p-TsCl, pyridine

2. Nal, acetone
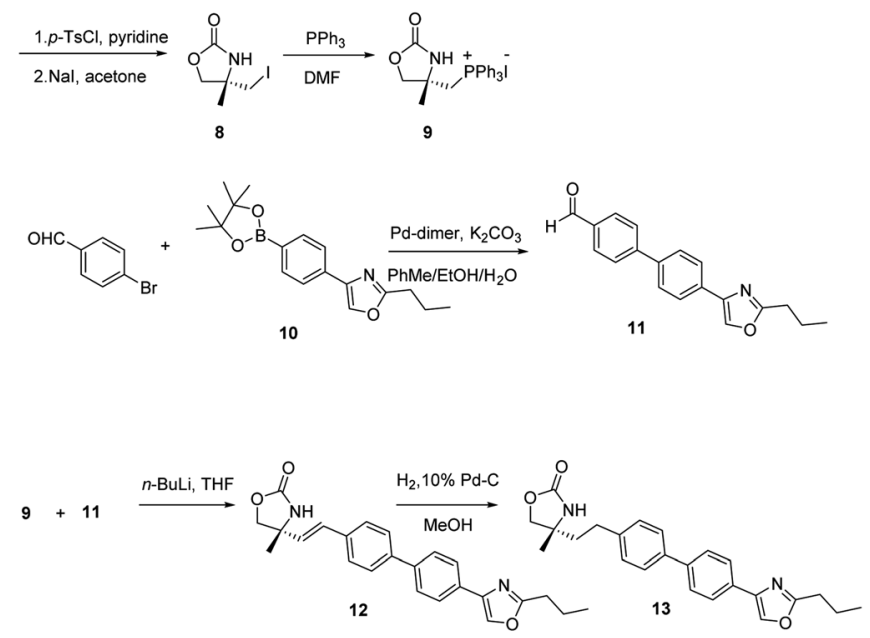

1. $\mathrm{KOH}, \mathrm{MeOH} / \mathrm{H}_{2} \mathrm{O} O \mathrm{OH} \mathrm{NH}_{2} \mathrm{HCl}$

2. ${\mathrm{HCl} / \mathrm{Et}_{2} \mathrm{O}}$

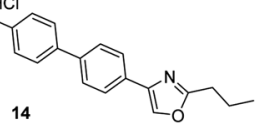

Scheme 1 Synthesis of the $\alpha$-substituted alaninol compound as S1P agonist.

29.7, 61.2, 62.5, 71.4, 71.8, 73.2, 127.8, 128.1, 128.7, 137.4, 156.3, 156.7; HRMS calcd for $\mathrm{C}_{15} \mathrm{H}_{21} \mathrm{~N}_{2} \mathrm{O}_{5}[\mathrm{M}+\mathrm{H}]^{+}$309.1445, found 309.1442; HPLC (Daicel Chiralpak OD-H, hexane/isopropanol = $90: 10$, flow rate $\left.1.0 \mathrm{~mL} \min ^{-1}, \lambda=254 \mathrm{~nm}\right): t_{\mathrm{R}}=16.37 \mathrm{~min}$ (major), $t_{\mathrm{R}}=20.63 \mathrm{~min}$ (minor), $57 \%$ ee.

\section{3-Benzyloxycarbonylamino-4-methyl-4-benzyloxy-oxazolidin-2-one} (5a-Bn)

Oil, yield 81\%; ${ }^{1} \mathrm{H}$ NMR (400 $\left.\mathrm{MHz}, \mathrm{CDCl}_{3}\right) \delta 1.26\left(\mathrm{~s}, 3 \mathrm{H}, \mathrm{CH}_{3}\right.$ ), 3.27 (d, $1 \mathrm{H}, J=12.0 \mathrm{~Hz}, \mathrm{CH}), 3.44$ (d, $1 \mathrm{H}, J=12.0 \mathrm{~Hz}, \mathrm{CH}), 4.06$ $(\mathrm{d}, 1 \mathrm{H}, J=12.0 \mathrm{~Hz}, \mathrm{CH}), 4.32(\mathrm{~d}, 1 \mathrm{H}, J=12.0 \mathrm{~Hz}, \mathrm{CH}), 4.46$ (s, $2 \mathrm{H}, \mathrm{CH}_{2}$ ), 5.14 (s, 2H, $\mathrm{CH}_{2}$ ), 6.38 (bs, 1H, NH), 7.24-7.35 (m, $\left.10 \mathrm{H}, \mathrm{H}_{\mathrm{ar}}\right) ;{ }^{13} \mathrm{C} \mathrm{NMR}\left(100 \mathrm{MHz}, \mathrm{CDCl}_{3}\right) \delta 19.8,61.2,68.1,71.4$, 71.7, 73.2, 127.8, 128.2, 128.3, 128.5, 128.6, 128.7, 135.3, 127.3, 156.1, 156.7; HRMS calcd for $\mathrm{C}_{20} \mathrm{H}_{23} \mathrm{~N}_{2} \mathrm{O}_{5}[\mathrm{M}+\mathrm{H}]^{+}$371.1602, found 371.1591; HPLC (Daicel Chiralpak OD, hexane/ isopropanol $=90: 10$, flow rate $1.0 \mathrm{~mL} \mathrm{~min}^{-1}, \lambda=254 \mathrm{~nm}$ ): $t_{\mathrm{R}}=17.15 \min$ (major), $t_{\mathrm{R}}=25.28 \mathrm{~min}$ (minor) $69 \%$ ee.

\section{3-(4-Chloro)benzyloxycarbonylamino-4-methyl-4-benzyloxy- oxazolidin-2-one (5a-p-ClBn)}

Catalyst 1d (64 mg, $15 \mathrm{~mol} \%$ in respect to the azodicarboxylate) was added to a suspension of 3-(benzyloxy)-2-methylpropanal (1.15 g, $6.46 \mathrm{mmol})$ and di-p-chlorobenzyl azodicarboxylate (1.58 g, 4.31 $\mathrm{mmol})$ in THF (40 mL). The mixture stirred at rt under argon until the colour of the azodicarboxylate had disappeared and quenched by the addition $\mathrm{H}_{2} \mathrm{O}$, then extracted three times with $\mathrm{Et}_{2} \mathrm{O}(50 \mathrm{ml}$ $\times 3$ ). The combined organic layers were dried over $\mathrm{Na}_{2} \mathrm{SO}_{4}$, filtered, and concentrated. The resulting crude was purified by flash chromatography on silica gel, eluting with light petroleum ether-ethyl acetate mixture ( $4: 1 \mathrm{v} / \mathrm{v}$ ) to afford $4 \mathrm{a}-\boldsymbol{p}$-ClBn $(1.87 \mathrm{~g})$ as solid in $80 \%$ yield with $71 \%$ ee. Recrystallization from $90 \%$ ethanol, the aldehyde 4a-p-ClBn (930 mg) was obtained in 97\% ee (50\% yield). $[\alpha]_{\mathrm{D}}^{20} 9.72(c$ $\left.0.29, \mathrm{CHCl}_{3}\right) .{ }^{1} \mathrm{H}$ NMR $\left(400 \mathrm{MHz}, \mathrm{CDCl}_{3}\right) \delta 1.34\left(\mathrm{~s}, 3 \mathrm{H}, \mathrm{CH}_{3}\right), 3.60-$ $3.77\left(\mathrm{~m}, 2 \mathrm{H}, \mathrm{CH}_{2}\right), 4.42\left(\mathrm{~s}, 2 \mathrm{H}, \mathrm{CH}_{2}\right), 5.01-5.15\left(\mathrm{~m}, 4 \mathrm{H}, 2 \mathrm{CH}_{2}\right), 6.70$ (s, 1H, NH), 7.16-7.30 (m, 13H, $\left.\mathrm{H}_{\mathrm{ar}}\right), 9.55$ (s, 1H, CHO); HRMS calcd for $\mathrm{C}_{27} \mathrm{H}_{27} \mathrm{~N}_{2} \mathrm{O}_{6} \mathrm{Cl}_{2}[\mathrm{M}+\mathrm{H}]^{+}$545.12407, found 545.12390; HPLC (Daicel Chiralpak AD-H, hexane/isopropanol $=85: 15$, flow rate 1.0 $\mathrm{mL} \min ^{-1}, \lambda=213 \mathrm{~nm}$ ): $t_{\mathrm{R}}=21.62 \mathrm{~min}$ (major), $t_{\mathrm{R}}=23.77 \mathrm{~min}$ (minor).

$\mathrm{NaBH}_{4}$ (190 mg, $5.0 \mathrm{mmol}$ ) was added to a solution of 4a-p-ClBn (900 mg, $1.65 \mathrm{mmol}$ ) in $\mathrm{CH}_{2} \mathrm{Cl}_{2} / \mathrm{C}_{2} \mathrm{H}_{5} \mathrm{OH}(4 \mathrm{~mL}$ ). The reaction mixture was stirred for $1 \mathrm{~h}$, and then it was quenched by adding $1 \mathrm{M}$ $\mathrm{HCl}$ aq. until the mixture reached $\mathrm{pH} 7$, and it was extracted with $\mathrm{CH}_{2} \mathrm{Cl}_{2}$. The combined organic phases were dried over $\mathrm{Na}_{2} \mathrm{SO}_{4}$, and the solvent was evaporated under reduced pressure. The resulting crude was purified by flash chromatography on silica gel eluted with light petroleum ether-ethyl acetate mixture (4:1 v/v) to afford 5a-pClBn (650 mg) in 94\% yield with $>99 \%$ ee. As oil; $[\alpha]_{\mathrm{D}}^{20}-12.3(c 0.13$, $\left.\mathrm{CHCl}_{3}\right) ;{ }^{1} \mathrm{H}$ NMR $\left(400 \mathrm{MHz}, \mathrm{CDCl}_{3}\right) \delta 1.24\left(\mathrm{~s}, 3 \mathrm{H}, \mathrm{CH}_{3}\right), 3.26(\mathrm{~d}, 1 \mathrm{H}, J$ $=8.0 \mathrm{~Hz}, \mathrm{CH}), 3.43(\mathrm{~d}, 1 \mathrm{H}, J=12.0 \mathrm{~Hz}, \mathrm{CH}), 4.05(\mathrm{~d}, 1 \mathrm{H}, J=8.0 \mathrm{~Hz}$, $\mathrm{CH}), 4.32(\mathrm{~d}, 1 \mathrm{H}, J=8.0 \mathrm{~Hz}, \mathrm{CH}), 4.49\left(\mathrm{~s}, 2 \mathrm{H}, \mathrm{CH}_{2}\right), 5.06\left(\mathrm{~s}, 2 \mathrm{H}, \mathrm{CH}_{2}\right)$, 6.49 (bs, $1 \mathrm{H}, \mathrm{NH}), 7.24-7.36\left(\mathrm{~m}, 10 \mathrm{H}, \mathrm{H}_{\mathrm{ar}}\right) ;{ }^{13} \mathrm{C} \mathrm{NMR}(100 \mathrm{MHz}$, $\left.\mathrm{CDCl}_{3}\right) \delta 19.8,61.2,67.2,71.4,71.7,73.2,127.8,128.2$, 128.5, 128.7, 128.8, 129.6, 134.0, 134.3, 137.3, 156.0, 156.7; HRMS calcd for $\mathrm{C}_{20} \mathrm{H}_{22} \mathrm{~N}_{2} \mathrm{O}_{5} \mathrm{Cl}[\mathrm{M}+\mathrm{H}]^{+}$405.1212, found 405.1204; HPLC (Daicel Chiralpak OD-H, hexane/isopropanol $=90: 10$, flow rate 1.0 $\mathrm{mL} \min ^{-1}, \lambda=213 \mathrm{~nm}$ ): $t_{\mathrm{R}}=27.77 \mathrm{~min}$ (major), $t_{\mathrm{R}}=35.0 \mathrm{~min}$ (minor).

\section{3-(4-Chloro)benzyloxycarbonylamino-4-methyl-4-(4-methyl)} benzyloxy-oxazolidin-2-one (5b-Bn)

Oil, yield 54\%; ${ }^{1} \mathrm{H}$ NMR (400 MHz, $\mathrm{CDCl}_{3}$ ) $\delta 1.24\left(\mathrm{~s}, 3 \mathrm{H}, \mathrm{CH}_{3}\right.$ ), $2.29\left(\mathrm{~s}, 3 \mathrm{H}, \mathrm{CH}_{3}\right), 3.24(\mathrm{~d}, 1 \mathrm{H}, J=12.0 \mathrm{~Hz}, \mathrm{CH}), 3.40$ (d, $1 \mathrm{H}, J=$ $12.0 \mathrm{~Hz}, \mathrm{CH}$ ), 3.99 (d, $1 \mathrm{H}, J=8.0 \mathrm{~Hz}, \mathrm{CH}), 4.31$ (d, $1 \mathrm{H}, J=8.0 \mathrm{~Hz}$, $\mathrm{CH})$, 4.40-4.48 (m, 2H, $\left.\mathrm{CH}_{2}\right), 5.12\left(\mathrm{~s}, 2 \mathrm{H}, \mathrm{CH}_{2}\right), 6.39$ (bs, $1 \mathrm{H}$, $\mathrm{NH}), 7.13-7.18\left(\mathrm{~m}, 4 \mathrm{H}, \mathrm{H}_{\mathrm{ar}}\right), 7.31-7.37\left(\mathrm{~m}, 5 \mathrm{H}, \mathrm{H}_{\mathrm{ar}}\right) ;{ }^{13} \mathrm{C} \mathrm{NMR}$ $\left(100 \mathrm{MHz}, \mathrm{CDCl}_{3}\right) \delta 19.8,21.2,61.2,68.0,71.3,71.4,73.0,73.1$, 128.1 , 128.2, 128.5, 128.6, 129.2, 129.4, 134.3, 135.4, 138.0, 156.2, 156.7; HRMS calcd for $\mathrm{C}_{21} \mathrm{H}_{25} \mathrm{~N}_{2} \mathrm{O}_{5}[\mathrm{M}+\mathrm{H}]^{+} 385.1758$, found 385.1738; HPLC (Daicel Chiralpak AD-H, hexane/ isopropanol $=90: 10$, flow rate $1.0 \mathrm{~mL} \mathrm{~min}^{-1}, \lambda=254 \mathrm{~nm}$ ): $t_{\mathrm{R}}=22.42 \min$ (major), $t_{\mathrm{R}}=25.10 \min$ (minor), $48 \%$ ee.

3-(4-Chloro)benzyloxycarbonylamino-4-methyl-4-(4-methyl) benzyloxy-oxazolidin-2-one ( $5 \mathrm{~b}-\boldsymbol{p}$-ClBn)

Oil, yield 56\%; ${ }^{1} \mathrm{H}$ NMR (400 MHz, $\mathrm{CDCl}_{3}$ ) $\delta 1.23\left(\mathrm{~s}, 3 \mathrm{H}, \mathrm{CH}_{3}\right), 2.30$ $\left(\mathrm{s}, 3 \mathrm{H}, \mathrm{CH}_{3}\right), 3.24$ (d, $\left.1 \mathrm{H}, J=12.0 \mathrm{~Hz}, \mathrm{CH}\right), 3.39$ (d, $1 \mathrm{H}, J=12.0 \mathrm{~Hz}$, $\mathrm{CH}), 4.04(\mathrm{~d}, 1 \mathrm{H}, J=8.0 \mathrm{~Hz}, \mathrm{CH}), 4.31$ (d, $1 \mathrm{H}, J=12.0 \mathrm{~Hz}, \mathrm{CH}), 4.39-$ $4.49\left(\mathrm{~m}, 2 \mathrm{H}, \mathrm{CH}_{2}\right), 5.08$ (s, 2H, $\left.\mathrm{CH}_{2}\right), 6.22(\mathrm{bs}, 1 \mathrm{H}, \mathrm{NH}), 7.13-7.16(\mathrm{~m}$, 
$\left.4 \mathrm{H}, \mathrm{H}_{\mathrm{ar}}\right), 7.26\left(\mathrm{~d}, 2 \mathrm{H}, J=8.0 \mathrm{~Hz}, \mathrm{H}_{\mathrm{ar}}\right), 7.32\left(\mathrm{~d}, 2 \mathrm{H}, J=8.0 \mathrm{~Hz}, \mathrm{H}_{\mathrm{ar}}\right)$; ${ }^{13} \mathrm{C}$ NMR $\left(100 \mathrm{MHz}, \mathrm{CDCl}_{3}\right) \delta 19.8,21.2,61.2,67.2,71.2,71.4,73.0$, 128.1, 128.8, 129.4, 129.6, 133.9, 134.2, 134.4, 138.1, 156.0, 156.7; HRMS calcd for $\mathrm{C}_{21} \mathrm{H}_{24} \mathrm{~N}_{2} \mathrm{O}_{5} \mathrm{Cl}[\mathrm{M}+\mathrm{H}]^{+}$419.1368, found 419.1359; HPLC (Daicel Chiralpak AD-H, hexane/isopropanol $=90: 10$, flow rate $1.0 \mathrm{~mL} \min ^{-1}, \lambda=254 \mathrm{~nm}$ ): $t_{\mathrm{R}}=23.38 \mathrm{~min}$ (major), $t_{\mathrm{R}}=$ $26.35 \mathrm{~min}$ (minor), $54 \%$ ee.

\section{3-Benzyloxycarbonylamino-4-methyl-4-(3,4-dimethoxy) benzyloxy-oxazolidin-2-one (5c-Et)}

Oil, yield 78\%; ${ }^{1} \mathrm{H}$ NMR (400 MHz, $\left.\mathrm{CDCl}_{3}\right) \delta 1.20\left(\mathrm{~m}, 6 \mathrm{H}, 2 \mathrm{CH}_{3}\right)$, $3.21(\mathrm{~d}, 1 \mathrm{H}, J=8.0 \mathrm{~Hz}, \mathrm{CH}), 3.40$ (d, $1 \mathrm{H}, J=8.0 \mathrm{~Hz}, \mathrm{CH}), 3.82$ (s, $\left.3 \mathrm{H}, \mathrm{CH}_{3}\right), 3.84\left(\mathrm{~s}, 3 \mathrm{H}, \mathrm{CH}_{3}\right), 4.01(\mathrm{~d}, 1 \mathrm{H}, J=8.0 \mathrm{~Hz}, \mathrm{CH}), 4.08-$ $4.13\left(\mathrm{~m}, 2 \mathrm{H}, \mathrm{CH}_{2}\right), 4.28(\mathrm{~d}, 1 \mathrm{H}, J=8.0 \mathrm{~Hz}, \mathrm{CH}), 4.37-4.44(\mathrm{~m}, 2 \mathrm{H}$, $\mathrm{CH}_{2}$ ), 6.45 (bs, $\left.1 \mathrm{H}, \mathrm{NH}\right), 6.76-6.80\left(\mathrm{~m}, 3 \mathrm{H}, \mathrm{H}_{\mathrm{ar}}\right) ;{ }^{13} \mathrm{C} \mathrm{NMR}(100$ $\left.\mathrm{MHz}, \mathrm{CDCl}_{3}\right) \delta 14.3,14.5,19.7,55.8,55.9,61.2,62.4,71.3,71.4$, 72.9, 110.9, 111.0, 120.3, 130.0, 148.9, 149.3, 156.3, 156.9; HRMS calcd for $\mathrm{C}_{17} \mathrm{H}_{24} \mathrm{~N}_{2} \mathrm{O}_{7} \mathrm{Na}[\mathrm{M}+\mathrm{Na}]^{+} 391.1476$, found 391.1471; HPLC (Daicel Chiralpak OD-H, hexane/isopropanol $=90: 10$,

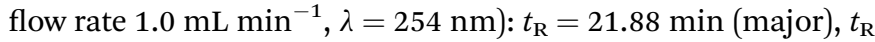
$=27.05 \mathrm{~min}$ (minor), $38 \%$ ee.

\section{3-Benzyloxycarbonylamino-4-methyl-4-(3,4-dimethoxy) benzyloxy-oxazolidin-2-one (5c-Bn)}

Oil, yield 67\%; ${ }^{1} \mathrm{H}$ NMR (400 MHz, $\mathrm{CDCl}_{3}$ ) $\delta 1.22\left(\mathrm{~s}, 3 \mathrm{H}, \mathrm{CH}_{3}\right.$ ), 3.22 $\left(\mathrm{d}, 1 \mathrm{H}, J=8.0 \mathrm{~Hz}, \mathrm{CH}_{2}\right), 3.40\left(\mathrm{~d}, 1 \mathrm{H}, J=12.0 \mathrm{~Hz}, \mathrm{CH}_{2}\right), 3.80(\mathrm{~s}, 3 \mathrm{H}$, $\left.\mathrm{CH}_{3}\right), 3.84\left(\mathrm{~s}, 3 \mathrm{H}, \mathrm{CH}_{3}\right), 4.03(\mathrm{~d}, 1 \mathrm{H}, J=8.0 \mathrm{~Hz}, \mathrm{CH}), 4.30(\mathrm{~d}, 1 \mathrm{H}, J=$ $8.0 \mathrm{~Hz}, \mathrm{CH}), 4.41\left(\mathrm{~m}, 2 \mathrm{H}, \mathrm{CH}_{2}\right), 5.11\left(\mathrm{~s}, 2 \mathrm{H}, \mathrm{CH}_{2}\right), 6.347$ (bs, 1H, NH), 6.79-6.82 (m, 3H, $\left.\mathrm{H}_{\mathrm{ar}}\right), 7.25-7.35\left(\mathrm{~m}, 5 \mathrm{H}, \mathrm{H}_{\mathrm{ar}}\right) ;{ }^{13} \mathrm{C} \mathrm{NMR}(100 \mathrm{MHz}$, $\left.\mathrm{CDCl}_{3}\right) \delta 19.7,55.9,61.3,68.0,71.3,71.4,72.9,111.0,120.3,128.2$, 128.4, 128.6, 129.9, 135.4, 148.9, 149.3, 156.2, 156.9; HRMS calcd for $\mathrm{C}_{22} \mathrm{H}_{26} \mathrm{~N}_{2} \mathrm{O}_{7} \mathrm{Na}[\mathrm{M}+\mathrm{H}]^{+}$453.1632, found 453.1638; HPLC (Daicel Chiralpak $\mathrm{AD}-\mathrm{H}$, hexane/isopropanol $=85: 15$, flow rate 1.0 $\mathrm{mL} \min ^{-1}, \lambda=254 \mathrm{~nm}$ ): $t_{\mathrm{R}}=26.31 \mathrm{~min}$ (major), $t_{\mathrm{R}}=32.42 \mathrm{~min}$ (minor), $45 \%$ ee.

\section{3-Ethyloxycarbonylamino-4-methyl-4-(4-trifluoro)benzyloxy- oxazolidin-2-one (5d-Et)}

White solid, yield 75\%; ${ }^{1} \mathrm{H}$ NMR (400 $\left.\mathrm{MHz}, \mathrm{CDCl}_{3}\right) \delta 1.21-1.26(\mathrm{~m}$, $6 \mathrm{H}, 2 \mathrm{CH}_{3}$ ), $3.26(\mathrm{~d}, 1 \mathrm{H}, J=12.0 \mathrm{~Hz}, \mathrm{CH}), 3.46(\mathrm{~d}, 1 \mathrm{H}, J=8.0 \mathrm{~Hz}$, $\mathrm{CH}), 4.04(\mathrm{~d}, 1 \mathrm{H}, J=8.0 \mathrm{~Hz}, \mathrm{CH}), 4.13\left(\mathrm{~m}, 2 \mathrm{H}, \mathrm{CH}_{2}\right), 4.30(\mathrm{~d}, 1 \mathrm{H}, J=$ 12.0 Hz, CH), 4.43-4.50 (m, 2H, $\mathrm{CH}_{2}$ ), 6.61 (bs, $\left.1 \mathrm{H}, \mathrm{NH}\right), 6.70-7.04$ $\left(\mathrm{m}, 2 \mathrm{H}, \mathrm{H}_{\mathrm{ar}}\right), 7.22-7.26\left(\mathrm{~m}, 2 \mathrm{H}, \mathrm{H}_{\mathrm{ar}}\right) ;{ }^{13} \mathrm{C} \mathrm{NMR}\left(100 \mathrm{MHz}, \mathrm{CDCl}_{3}\right)$ $\delta 14.3,19.7,61.2,62.5,71.4,71.9,72.5,115.4,115.6,129.4,129.5$, 133.2, 156.4, 156.8, 161.3, 163.7; HRMS calcd for $\mathrm{C}_{15} \mathrm{H}_{20} \mathrm{~N}_{2} \mathrm{O}_{5} \mathrm{~F}[\mathrm{M}+$ $\mathrm{H}]^{+}$327.1351, found 327.1341; HPLC (Daicel Chiralpak AS-H, hexane/isopropanol $=80: 20$, flow rate $1.0 \mathrm{~mL} \mathrm{~min}^{-1}, \lambda=254$ $\mathrm{nm}$ ): $t_{\mathrm{R}}=51.09 \min$ (major), $t_{\mathrm{R}}=76.63 \mathrm{~min}$ (minor), $56 \%$ ee.

\section{3-Benzyloxycarbonylamino-4-methyl-4-(4-fluoro)benzyloxy- oxazolidin-2-one (5d-Bn)}

Oil, yield 80\%; ${ }^{1} \mathrm{H}$ NMR (400 MHz, $\left.\mathrm{CDCl}_{3}\right) \delta 1.33\left(\mathrm{~s}, 3 \mathrm{H}, \mathrm{CH}_{3}\right.$ ), 3.32 (d, $1 \mathrm{H}, J=8.0 \mathrm{~Hz}, \mathrm{CH}), 3.51$ (d, $1 \mathrm{H}, J=8.0 \mathrm{~Hz}, \mathrm{CH}), 4.12$ (d, $1 \mathrm{H}, J=4.0 \mathrm{~Hz}, \mathrm{CH}), 4.37$ (d, $1 \mathrm{H}, J=8.0 \mathrm{~Hz}, \mathrm{CH}), 4.51(\mathrm{~s}, 2 \mathrm{H}$, $\mathrm{CH}_{2}$ ), 5.21 (s, 2H, $\mathrm{CH}_{2}$ ), 6.42 (bs, $\left.1 \mathrm{H}, \mathrm{NH}\right), 7.08$ (t, $J=4.0 \mathrm{~Hz}, 2 \mathrm{H}$,
$\mathrm{H}_{\mathrm{ar}}$ ), $7.30\left(\mathrm{~d}, 2 \mathrm{H}, J=8.0 \mathrm{~Hz}, \mathrm{H}_{\mathrm{ar}}\right.$ ), 7.40 (bs, 5H, $\left.\mathrm{H}_{\mathrm{ar}}\right) ;{ }^{13} \mathrm{C} \mathrm{NMR}$ $\left(100 \mathrm{MHz}, \mathrm{CDCl}_{3}\right) \delta 19.8,60.4,68.1,71.4,71.9,72.5,115.7$, 128.2 , 128.5, 128.6, 129.4, 129.5, 132.5, 133.2, 135.2, 156.2, 156.7; HRMS calcd for $\mathrm{C}_{20} \mathrm{H}_{22} \mathrm{~N}_{2} \mathrm{O}_{5} \mathrm{~F}[\mathrm{M}+\mathrm{H}]^{+} 389.1507$, found 389.1502; HPLC (Daicel Chiralpak AD-H, hexane/isopropanol =

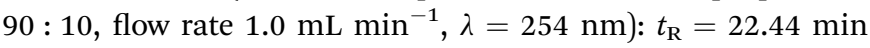
(major), $t_{\mathrm{R}}=24.32 \mathrm{~min}$ (minor), 68\% ee.

3-(4-Chloro)benzyloxycarbonylamino-4-methyl-4-(4-fluoro) benzyloxy-oxazolidin-2-one (5d-p-ClBn)

Oil, yield 89\%; ${ }^{1} \mathrm{H}$ NMR (400 MHz, $\mathrm{CDCl}_{3}$ ) $\delta 1.24\left(\mathrm{~s}, 3 \mathrm{H}, \mathrm{CH}_{3}\right), 3.24$ $(\mathrm{d}, 1 \mathrm{H}, J=8.0 \mathrm{~Hz}, \mathrm{CH}), 3.43$ (d, $1 \mathrm{H}, J=12.0 \mathrm{~Hz}, \mathrm{CH}), 4.04$ (d, 1H, $J=$ $8.0 \mathrm{~Hz}, \mathrm{CH}), 4.30$ (d, $1 \mathrm{H}, J=8.0 \mathrm{~Hz}, \mathrm{CH}), 4.40-4.48\left(\mathrm{~m}, 2 \mathrm{H}, \mathrm{CH}_{2}\right)$, 5.07 (s, 2H, $\mathrm{CH}_{2}$ ), 6.84 (bs, $\left.1 \mathrm{H}, \mathrm{NH}\right), 7.00$ (t, 2H, $J=8.0 \mathrm{~Hz}, \mathrm{H}_{\mathrm{ar}}$ ), 7.21-7.25 (m, 2H, $\left.\mathrm{H}_{\mathrm{ar}}\right), 7.30\left(\mathrm{~d}, 2 \mathrm{H}, J=12.0 \mathrm{~Hz}, \mathrm{H}_{\mathrm{ar}}\right) ;{ }^{13} \mathrm{C} \mathrm{NMR}(100$ $\left.\mathrm{MHz}, \mathrm{CDCl}_{3}\right) \delta$ 19.7, 61.2, 67.3, 71.4, 71.8, 72.5, 115.4, 115.7, 128.8, 129.0, 129.4, 129.5, 133.1, 133.2, 134.0, 134.2, 156.1, 156.8, 161.3, 163.7; HRMS calcd for $\mathrm{C}_{20} \mathrm{H}_{21} \mathrm{~N}_{2} \mathrm{O}_{5} \mathrm{ClF}[\mathrm{M}+\mathrm{H}]^{+} 423.1118$, found 423.1104; HPLC (Daicel Chiralpak AD-H, hexane/isopropanol =

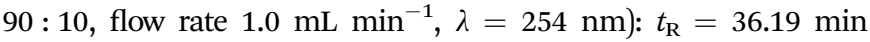
(major), $t_{\mathrm{R}}=45.33 \mathrm{~min}$ (minor), $70 \%$ ee.

3-Ethyloxycarbonylamino-4-methyl-4-(4-chloro)benzyloxyoxazolidin-2-one (5e-Et)

White solid, yield 78\%, mp 80-85 ${ }^{\circ} \mathrm{C} ;{ }^{1} \mathrm{H}$ NMR (400 MHz, $\mathrm{CDCl}_{3}$ ) $\delta 1.25\left(\mathrm{~m}, 6 \mathrm{H}, 2 \mathrm{CH}_{3}\right), 3.27$ (d, $\left.1 \mathrm{H}, J=8.0 \mathrm{~Hz}, \mathrm{CH}\right), 3.48$ (d, $1 \mathrm{H}, J=$ $12.0 \mathrm{~Hz}, \mathrm{CH}), 4.06$ (d, $1 \mathrm{H}, J=8.0 \mathrm{~Hz}, \mathrm{CH}), 4.17$ (q, $2 \mathrm{H}, J=16 \mathrm{~Hz}$, $\left.8 \mathrm{~Hz}, \mathrm{CH}_{2}\right), 4.32(\mathrm{~d}, 1 \mathrm{H}, J=8.0 \mathrm{~Hz}, \mathrm{CH}), 4.47-4.48\left(\mathrm{~m}, 2 \mathrm{H}, \mathrm{CH}_{2}\right), 6.39$ (bs, 1H, NH), 6.76-6.80 (m, 4H, Har); ${ }^{13} \mathrm{C} \mathrm{NMR} \mathrm{(100} \mathrm{MHz,} \mathrm{CDCl}_{3}$ ) $\delta$ 14.3, 19.8, 61.2, 62.6, 71.4, 72.1, 72.5, 128.8, 129.0, 133.9, 135.9, 156.3, 156.7; HRMS calcd for $\mathrm{C}_{15} \mathrm{H}_{20} \mathrm{~N}_{2} \mathrm{O}_{5} \mathrm{Cl}[\mathrm{M}+\mathrm{H}]^{+} 343.1055$, found 343.1048; HPLC (Daicel Chiralpak OJ-H, hexane/isopropanol $=90: 10$, flow rate $\left.1.0 \mathrm{~mL} \min ^{-1}, \lambda=254 \mathrm{~nm}\right): t_{\mathrm{R}}=32.27 \mathrm{~min}$ (major), $t_{\mathrm{R}}=37.97 \mathrm{~min}$ (minor), $57 \%$ ee.

\section{3-Benzyloxycarbonylamino-4-methyl-4-(4-chloro)benzyloxy-} oxazolidin-2-one (5e-Bn)

White solid, yield $63 \%$; $\mathrm{mp} 75-80{ }^{\circ} \mathrm{C} ;{ }^{1} \mathrm{H} \mathrm{NMR}\left(400 \mathrm{MHz}, \mathrm{CDCl}_{3}\right.$ ) $\delta 1.26\left(\mathrm{~s}, 3 \mathrm{H}, \mathrm{CH}_{3}\right), 3.25$ (d, $\left.1 \mathrm{H}, J=8.0 \mathrm{~Hz}, \mathrm{CH}\right), 3.45$ (d, $1 \mathrm{H}, J=$ $8.0 \mathrm{~Hz}, \mathrm{CH}), 4.06$ (d, $1 \mathrm{H}, J=8.0 \mathrm{~Hz}, \mathrm{CH}), 4.31$ (d, $1 \mathrm{H}, J=8.0 \mathrm{~Hz}$, $\mathrm{CH})$, 4.41-4.49 (m, 2H, $\left.\mathrm{CH}_{2}\right), 5.13\left(\mathrm{~s}, 2 \mathrm{H}, \mathrm{CH}_{2}\right), 6.60(\mathrm{bs}, 1 \mathrm{H}$, $\mathrm{NH}), 7.19\left(\mathrm{~d}, 2 \mathrm{H}, J=8.0 \mathrm{~Hz}, \mathrm{H}_{\mathrm{ar}}\right), 7.29-7.37\left(\mathrm{~m}, 7 \mathrm{H}, \mathrm{H}_{\mathrm{ar}}\right) ;{ }^{13} \mathrm{C}$ NMR (100 MHz, $\mathrm{CDCl}_{3}$ ) $\delta 19.8,60.4,61.2,68.1,71.4,71.7,72.1$, 72.5, 127.8, 128.2, 128.5, 128.7, 128.8, 129.0, 133.9, 135.3, 135.9, 156.2, 156.7; HRMS calcd for $\mathrm{C}_{20} \mathrm{H}_{22} \mathrm{~N}_{2} \mathrm{O}_{5} \mathrm{Cl}[\mathrm{M}+\mathrm{H}]^{+}$405.1212, found 405.1201; HPLC (Daicel Chiralpak AD-H, hexane/ isopropanol $=90: 10$, flow rate $\left.1.0 \mathrm{~mL} \mathrm{~min}^{-1}, \lambda=254 \mathrm{~nm}\right)$ : $t_{\mathrm{R}}=16.12 \min$ (major), $t_{\mathrm{R}}=17.62 \min$ (minor), $59 \%$ ee.

\section{3-Benzyloxycarbonylamino-4-methyl-4-(4-bromo)benzyloxy-} oxazolidin-2-one (5f-Bn)

White solid, yield 79\%; mp 80-84 ${ }^{\circ} \mathrm{C} ;{ }^{1} \mathrm{H}$ NMR (400 $\mathrm{MHz}, \mathrm{CDCl}_{3}$ ) $\delta 1.33\left(\mathrm{~s}, 3 \mathrm{H}, \mathrm{CH}_{3}\right), 3.32(\mathrm{~d}, 1 \mathrm{H}, J=8.0 \mathrm{~Hz}, \mathrm{CH}), 3.52(\mathrm{~d}, 1 \mathrm{H}, J=$ $8.0 \mathrm{~Hz}, \mathrm{CH}), 4.12$ (d, $1 \mathrm{H}, J=8.0 \mathrm{~Hz}, \mathrm{CH}), 4.37$ (d, $1 \mathrm{H}, J=8.0 \mathrm{~Hz}$, $\mathrm{CH})$, 4.49-4.50 (m, 2H, $\left.\mathrm{CH}_{2}\right), 5.20\left(\mathrm{~s}, 2 \mathrm{H}, \mathrm{CH}_{2}\right), 6.49$ (bs, $\left.1 \mathrm{H}, \mathrm{NH}\right)$, 
$7.19\left(\mathrm{~d}, 2 \mathrm{H}, J=4.0 \mathrm{~Hz}, \mathrm{H}_{\mathrm{ar}}\right), 7.40\left(\mathrm{~m}, 5 \mathrm{H}, \mathrm{H}_{\mathrm{ar}}\right), 7.52(\mathrm{~d}, 2 \mathrm{H}, J=$ $\left.8.0 \mathrm{~Hz}, \mathrm{H}_{\mathrm{ar}}\right) ;{ }^{13} \mathrm{C} \mathrm{NMR}\left(100 \mathrm{MHz}, \mathrm{CDCl}_{3}\right) \delta 19.7,61.2,68.1,71.4,72.2$, 72.5, 121.9, 128.2, 128.5, 128.7, 129.3, 131.7, 135.4, 136.5, 156.3, 156.7; HRMS calcd for $\mathrm{C}_{20} \mathrm{H}_{22} \mathrm{~N}_{2} \mathrm{O}_{5} \mathrm{Br}[\mathrm{M}+\mathrm{H}]^{+} 449.0707$, found 449.0710; HPLC (Daicel Chiralpak AD-H, hexane/isopropanol = $85: 15$, flow rate $\left.1.0 \mathrm{~mL} \min ^{-1}, \lambda=254 \mathrm{~nm}\right): t_{\mathrm{R}}=19.46 \mathrm{~min}$ (major), $t_{\mathrm{R}}=21.85 \mathrm{~min}$ (minor), 59\% ee.

4-Chlorobenzyl(4-(((4-bromobenzyl)oxy)methyl)-4-methyl2-oxooxazolidin-3-yl)carbamate (5f-p-ClBn)

Oil, yield 75\%; ${ }^{1} \mathrm{H}$ NMR (400 $\left.\mathrm{MHz}, \mathrm{CDCl}_{3}\right) \delta 1.27\left(\mathrm{~s}, 3 \mathrm{H}, \mathrm{CH}_{3}\right)$, 3.27 (d, $1 \mathrm{H}, J=10 \mathrm{~Hz}, \mathrm{CH}), 3.45$ (d, $1 \mathrm{H}, J=10 \mathrm{~Hz}, \mathrm{CH}), 4.08$ (d, $1 \mathrm{H}, J=8.0 \mathrm{~Hz}, \mathrm{CH}), 4.32(\mathrm{~d}, 1 \mathrm{H}, J=8.0 \mathrm{~Hz}, \mathrm{CH}), 4.45(\mathrm{~s}, 2 \mathrm{H}$, $\left.\mathrm{CH}_{2}\right), 5.11\left(\mathrm{~s}, 2 \mathrm{H}, \mathrm{CH}_{2}\right), 6.27(\mathrm{bs}, 1 \mathrm{H}, \mathrm{NH}), 7.14(\mathrm{~d}, 2 \mathrm{H}, J=8.0 \mathrm{~Hz}$, $\mathrm{H}_{\mathrm{ar}}$ ), $7.27\left(\mathrm{~d}, 2 \mathrm{H}, J=8.0 \mathrm{~Hz}, \mathrm{H}_{\mathrm{ar}}\right), 7.33\left(\mathrm{~d}, 2 \mathrm{H}, J=8.0 \mathrm{~Hz}, \mathrm{H}_{\mathrm{ar}}\right.$ ), 7.47 (d, 2H, $\left.J=8.0 \mathrm{~Hz}, \mathrm{H}_{\mathrm{ar}}\right) ;{ }^{13} \mathrm{C}$ NMR $\left(100 \mathrm{MHz}, \mathrm{CDCl}_{3}\right) \delta 19.7$, $61.2,68.1,71.4,72.2,72.5,121.9,128.2,128.5,128.7,129.3$, 131.7, 135.4, 136.5, 156.3, 156.7; HRMS calcd for $\mathrm{C}_{20} \mathrm{H}_{21} \mathrm{~N}_{2} \mathrm{O}_{5}$ $\mathrm{ClBr}[\mathrm{M}+\mathrm{H}]^{+}$483.0317, found 483.0315; HPLC (Daicel Chiralpak $\mathrm{AD}-\mathrm{H}$, hexane/isopropanol $=85: 15$, flow rate 1.0 $\mathrm{mL} \min ^{-1}, \lambda=254 \mathrm{~nm}$ ): $t_{\mathrm{R}}=22.65 \mathrm{~min}$ (major), $t_{\mathrm{R}}=27.78 \mathrm{~min}$ (minor), 59\% ee.

\section{3-Ethyloxycarbonylamino-4-methyl-4-(4-cyano)benzyloxy- oxazolidin-2-one (5g-Et)}

Oil, yield 81\%; ${ }^{1} \mathrm{H}$ NMR (400 MHz, $\left.\mathrm{CDCl}_{3}\right) \delta 1.22\left(\mathrm{~m}, 6 \mathrm{H}, 2 \mathrm{CH}_{3}\right)$, 3.33 (d, $1 \mathrm{H}, J=8.0 \mathrm{~Hz}, \mathrm{CH}), 3.55$ (d, $1 \mathrm{H}, J=8.0 \mathrm{~Hz}, \mathrm{CH}), 4.06$ (d, $1 \mathrm{H}, J=4.0 \mathrm{~Hz}, \mathrm{CH}), 4.11-4.16\left(\mathrm{~m}, 2 \mathrm{H}, \mathrm{CH}_{2}\right), 4.34(\mathrm{~d}, 1 \mathrm{H}, J=$ 12.0 Hz, CH), 4.51-4.62 (m, 2H, $\mathrm{CH}_{2}$ ), 6.892 (bs, 1H, NH), 7.36 $\left(\mathrm{d}, 2 \mathrm{H}, J=8.0 \mathrm{~Hz}, \mathrm{H}_{\mathrm{ar}}\right), 7.58\left(\mathrm{~d}, 2 \mathrm{H}, J=8.0 \mathrm{~Hz}, \mathrm{H}_{\mathrm{ar}}\right) ;{ }^{13} \mathrm{C} \mathrm{NMR}$ $\left(100 \mathrm{MHz}, \mathrm{CDCl}_{3}\right) \delta 14.3,19.6,61.2,62.5,70.8,71.3,72.3,72.8$, $111.4,118.8,127.6,130.3,132.3$, 132.4, 143.1, 156.5, 156.7; HRMS calcd for $\mathrm{C}_{16} \mathrm{H}_{20} \mathrm{~N}_{3} \mathrm{O}_{5}[\mathrm{M}+\mathrm{H}]^{+} 334.1398$, found 334.1416; HPLC (Daicel Chiralpak AD-H, hexane/isopropanol $=90: 10$,

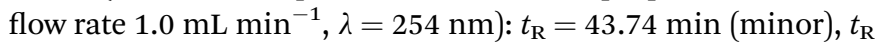
$=48.41 \mathrm{~min}$ (major), $57 \%$ ee.

\section{3-Benzyloxycarbonylamino-4-methyl-4-(4-cyano)benzyloxy- oxazolidin-2-one (5g-Bn)}

Oil, yield 71\%; ${ }^{1} \mathrm{H}$ NMR (400 MHz, $\left.\mathrm{CDCl}_{3}\right) \delta 1.27$ (s, 3H, $\mathrm{CH}_{3}$ ), 3.31 (d, $1 \mathrm{H}, J=8.0 \mathrm{~Hz}, \mathrm{CH}), 3.52$ (d, $1 \mathrm{H}, J=8.0 \mathrm{~Hz}, \mathrm{CH}), 4.05$ (d, $1 \mathrm{H}, J=8.0 \mathrm{~Hz}, \mathrm{CH}), 4.33(\mathrm{~d}, 1 \mathrm{H}, J=8.0 \mathrm{~Hz}, \mathrm{CH}), 4.48-4.58(\mathrm{~m}$, $\left.2 \mathrm{H}, \mathrm{CH}_{2}\right), 5.10\left(\mathrm{~s}, 2 \mathrm{H}, \mathrm{CH}_{2}\right), 7.09(\mathrm{bs}, 1 \mathrm{H}, \mathrm{NH}), 7.25-7.35(\mathrm{~m}, 7 \mathrm{H}$, $\mathrm{H}_{\mathrm{ar}}$ ), 7.55 (d, $\left.2 \mathrm{H}, J=8.0 \mathrm{~Hz}, \mathrm{H}_{\mathrm{ar}}\right) ;{ }^{13} \mathrm{C} \mathrm{NMR}\left(100 \mathrm{MHz}, \mathrm{CDCl}_{3}\right.$ ) $\delta$ 19.7, 61.2, 68.1, 71.4, 72.3, 72.9, 111.5, 118.7, 127.5, 128.2, 128.5, 128.7, 132.4, 135.3, 143.0, 156.3, 156.7; HRMS calcd for $\mathrm{C}_{21} \mathrm{H}_{22} \mathrm{~N}_{3} \mathrm{O}_{5}[\mathrm{M}+\mathrm{H}]^{+}$396.1554, found 396.1573; HPLC (Daicel Chiralpak $\mathrm{AD}-\mathrm{H}$, hexane/isopropanol $=90: 10$, flow rate 1.0 $\mathrm{mL} \min ^{-1}, \lambda=254 \mathrm{~nm}$ ): $t_{\mathrm{R}}=65.86 \min$ (major), $t_{\mathrm{R}}=69.48 \mathrm{~min}$ (minor), 65\% ee.

3-(4-Chloro)benzyloxycarbonylamino-4-methyl-4-(4-cyano) benzyloxy-oxazolidin-2-one (5g-p-ClBn)

Oil, yield 73\%; ${ }^{1} \mathrm{H}$ NMR (400 MHz, $\left.\mathrm{CDCl}_{3}\right) \delta 1.28\left(\mathrm{~s}, 3 \mathrm{H}, \mathrm{CH}_{3}\right)$, 3.31 (d, $1 \mathrm{H}, J=8.0 \mathrm{~Hz}, \mathrm{CH}), 3.50$ (d, $1 \mathrm{H}, J=12.0 \mathrm{~Hz}, \mathrm{CH}), 4.08$ $(\mathrm{d}, 1 \mathrm{H}, J=8.0 \mathrm{~Hz}, \mathrm{CH}), 4.34(\mathrm{~d}, 1 \mathrm{H}, J=8.0 \mathrm{~Hz}, \mathrm{CH}), 4.51-4.60$ (m, 2H, $\mathrm{CH}_{2}$ ), 5.09 (s, 2H, $\mathrm{CH}_{2}$ ), 6.62 (bs, 1H, NH), 7.25 (d, 2H, J $\left.=4.0 \mathrm{~Hz}, \mathrm{H}_{\mathrm{ar}}\right), 7.31\left(\mathrm{~d}, 2 \mathrm{H}, J=8.0 \mathrm{~Hz}, \mathrm{H}_{\mathrm{ar}}\right), 7.37$ (d, 2H, $J=$ $8.0 \mathrm{~Hz}, \mathrm{H}_{\mathrm{ar}}$ ), 7.59 (d, $\left.2 \mathrm{H}, J=8.0 \mathrm{~Hz}, \mathrm{H}_{\mathrm{ar}}\right) ;{ }^{13} \mathrm{C}$ NMR $(100 \mathrm{MHz}$, $\left.\mathrm{CDCl}_{3}\right) \delta 19.7,61.2,67.3,71.4,72.5,120.0,122.7,125.4,125.5$, 125.6, 125.7, 127.5, 128.9, 133.8, 134.5, 141.4, 156.1, 156.7; HRMS calcd for $\mathrm{C}_{21} \mathrm{H}_{21} \mathrm{~N}_{3} \mathrm{O}_{5} \mathrm{Cl}[\mathrm{M}+\mathrm{H}]^{+}$430.1164, found 430.1159; HPLC (Daicel Chiralpak AD-H, hexane/isopropanol = $85: 15$, flow rate $\left.1.0 \mathrm{~mL} \min ^{-1}, \lambda=254 \mathrm{~nm}\right): t_{\mathrm{R}}=50.46 \mathrm{~min}$ (major), $t_{\mathrm{R}}=57.77 \mathrm{~min}$ (minor), 62\% ee.

3-Ethyloxycarbonylamino-4-methyl-4-(4-trifluoro)benzyloxyoxazolidin-2-one (5h-Et)

White solid, yield 76\%; ${ }^{1} \mathrm{H}$ NMR (400 MHz, $\left.\mathrm{CDCl}_{3}\right) \delta 1.23(\mathrm{t}, 3 \mathrm{H}$, $\left.J=8.0 \mathrm{~Hz}, \mathrm{CH}_{3}\right), 1.29\left(\mathrm{~s}, 3 \mathrm{H}, \mathrm{CH}_{3}\right), 3.31(\mathrm{~d}, 1 \mathrm{H}, J=12.0 \mathrm{~Hz}, \mathrm{CH})$, $3.52(\mathrm{~d}, 1 \mathrm{H}, J=12.0 \mathrm{~Hz}, \mathrm{CH}), 4.06$ (d, $1 \mathrm{H}, J=8.0 \mathrm{~Hz}, \mathrm{CH}), 4.14$ (q, $\left.2 \mathrm{H}, J=12.0 \mathrm{~Hz}, 4 \mathrm{~Hz}, \mathrm{CH}_{2}\right), 4.34(\mathrm{~d}, 1 \mathrm{H}, J=8.0 \mathrm{~Hz}, \mathrm{CH}), 4.52-$ $4.62\left(\mathrm{~m}, 2 \mathrm{H}, \mathrm{CH}_{2}\right), 6.78$ (bs, $\left.1 \mathrm{H}, \mathrm{NH}\right), 7.38$ (d, $2 \mathrm{H}, J=8.0 \mathrm{~Hz}$, $\mathrm{H}_{\mathrm{ar}}$ ), $7.58\left(\mathrm{~d}, 2 \mathrm{H}, J=8.0 \mathrm{~Hz}, \mathrm{H}_{\mathrm{ar}}\right) ;{ }^{13} \mathrm{C} \mathrm{NMR}\left(100 \mathrm{MHz}, \mathrm{CDCl}_{3}\right.$ ) $\delta$ 14.3, 19.6, 61.2, 62.5, 71.3, 72.4, 72.5, 125.5, 127.5, 141.7, 156.4, 156.8; HRMS calcd for $\mathrm{C}_{16} \mathrm{H}_{20} \mathrm{~N}_{2} \mathrm{O}_{5} \mathrm{~F}_{3}[\mathrm{M}+\mathrm{H}]^{+}$377.1319, found 377.1315; HPLC (Daicel Chiralpak AD-H, hexane/ isopropanol $=90: 10$, flow rate $\left.1.0 \mathrm{~mL} \mathrm{~min}^{-1}, \lambda=254 \mathrm{~nm}\right)$ : $t_{\mathrm{R}}=13.40 \mathrm{~min}$ (minor), $t_{\mathrm{R}}=15.24 \mathrm{~min}$ (major), $56 \%$ ee.

\section{3-Benzyloxycarbonylamino-4-methyl-4-(4-trifluoro)benzyloxy- oxazolidin-2-one (5h-Bn)}

Oil, yield 89\%; ${ }^{1} \mathrm{H}$ NMR (400 MHz, $\left.\mathrm{CDCl}_{3}\right) \delta 1.27\left(\mathrm{~s}, 3 \mathrm{H}, \mathrm{CH}_{3}\right.$ ), 3.30 (d, $1 \mathrm{H}, J=12.0 \mathrm{~Hz}, \mathrm{CH}), 3.51$ (d, $1 \mathrm{H}, J=8.0 \mathrm{~Hz}, \mathrm{CH}), 4.05$ (d, $1 \mathrm{H}, J=8.0 \mathrm{~Hz}, \mathrm{CH}), 4.33(\mathrm{~d}, 1 \mathrm{H}, J=8.0 \mathrm{~Hz}, \mathrm{CH}), 4.49-4.59$ $\left(\mathrm{m}, 2 \mathrm{H}, \mathrm{CH}_{2}\right), 5.12\left(\mathrm{~s}, 2 \mathrm{H}, \mathrm{CH}_{2}\right), 6.87$ (bs, 1H, NH), 7.25-7.38 (m, $\left.7 \mathrm{H}, \mathrm{H}_{\mathrm{ar}}\right), 7.58\left(\mathrm{~d}, 2 \mathrm{H}, J=4.0 \mathrm{~Hz}, \mathrm{H}_{\mathrm{ar}}\right) ;{ }^{13} \mathrm{C} \mathrm{NMR}(100 \mathrm{MHz}$, $\left.\mathrm{CDCl}_{3}\right) \delta 19.7,61.2,68.1,71.4,72.5,72.6,125.5,125.6,127.4$, 128.2, 128.5, 128.6, 135.3, 141.6, 156.3, 156.8; HRMS calcd for $\mathrm{C}_{21} \mathrm{H}_{22} \mathrm{~N}_{2} \mathrm{O}_{5} \mathrm{~F}_{3}[\mathrm{M}+\mathrm{H}]^{+}$439.1475, found 439.1470; HPLC (Daicel Chiralpak AS-H, hexane/isopropanol $=80: 20$, flow rate 1.0 $\mathrm{mL} \min ^{-1}, \lambda=254 \mathrm{~nm}$ ): $t_{\mathrm{R}}=29.18 \mathrm{~min}$ (major), $t_{\mathrm{R}}=61.72 \mathrm{~min}$ (minor), 67\% ee.

\section{3-(4-Chloro)benzyloxycarbonylamino-4-methyl-4-(4-trifluoro)} benzyloxy-oxazolidin-2-one ( $5 \mathrm{~h}-\mathrm{p}$-ClBn)

Catalyst 1d (191 mg, $15 \mathrm{~mol} \%$ in respect to the azodicarboxylate) was added to a suspension of 2-methyl-3-((4-(trifluoromethyl) benzyl)oxy)propanal $(2.2 \mathrm{~g}, 8.93 \mathrm{mmol})$ and di-p-chlorobenzyl azodicarboxylate $(2.17 \mathrm{~g}, 5.93 \mathrm{mmol})$ in THF $(50 \mathrm{~mL})$. The mixture stirred at rt under argon until the colour of the azodicarboxylate had disappeared and quenched by the addition $\mathrm{H}_{2} \mathrm{O}$, then extracted three times with $\mathrm{Et}_{2} \mathrm{O}(50 \mathrm{~mL} \times 3)$. The combined organic layers were dried over $\mathrm{Na}_{2} \mathrm{SO}_{4}$, filtered, and concentrated. The resulting crude was purified by flash chromatography on silica gel, eluting with light petroleum etherethyl acetate mixture $(4: 1 \mathrm{v} / \mathrm{v})$ to afford $\mathbf{4 h}$ - $\boldsymbol{p}$-ClBn $(3.27 \mathrm{~g})$ as solid in $90 \%$ yield with $74 \%$ ee. Mp $145-149{ }^{\circ} \mathrm{C} ;{ }^{1} \mathrm{H}$ NMR (400 $\left.\mathrm{MHz} \mathrm{CDCl}_{3}\right) \delta 1.32\left(\mathrm{~s}, 3 \mathrm{H}, \mathrm{CH}_{3}\right), 3.71-3.87\left(\mathrm{~m}, 2 \mathrm{H}, \mathrm{CH}_{2}\right), 4.54(\mathrm{~s}$, $\left.2 \mathrm{H}, \mathrm{CH}_{2}\right), 5.10-5.20\left(\mathrm{~m}, 4 \mathrm{H}, 2 \mathrm{CH}_{2}\right), 6.71(\mathrm{~s}, 1 \mathrm{H}, \mathrm{NH}), 7.22-7.38$ (m, 10H, $\mathrm{H}_{\mathrm{ar}}$ ), 7.62 (d, 2H, $J=4.0 \mathrm{~Hz}, \mathrm{H}_{\mathrm{ar}}$ ), 9.62 (s, 1H, CHO); 
HRMS calcd for $\mathrm{C}_{28} \mathrm{H}_{26} \mathrm{~N}_{2} \mathrm{O}_{6} \mathrm{Cl}_{2} \mathrm{~F}_{3}[\mathrm{M}+\mathrm{H}]^{+}$613.1115, found 613.1110; HPLC (Daicel Chiralpak AD-H, hexane/isopropanol = $85: 15$, flow rate $\left.1.0 \mathrm{~mL} \min ^{-1}, \lambda=254 \mathrm{~nm}\right): t_{\mathrm{R}}=18.00 \mathrm{~min}$ (major), $t_{\mathrm{R}}=20.29 \mathrm{~min}$ (minor).

Upon recrystallization from $90 \%$ ethanol, the aldehyde $\mathbf{4 h}-\boldsymbol{p}$ ClBn (2.1 g) was obtained in 98\% ee (65\% yield). After reduction and cyclization with $\mathrm{NaBH}_{4}(380 \mathrm{mg}, 10 \mathrm{mmol}), \mathbf{5 h}-\boldsymbol{p}$-ClBn $(1.53$ g) was obtained in $95 \%$ yield with $98 \%$ ee. $[\alpha]_{\mathrm{D}}^{20}-17.84(c 0.7$, $\left.\mathrm{CHCl}_{3}\right) ;{ }^{1} \mathrm{H}$ NMR $\left(400 \mathrm{MHz}, \mathrm{CDCl}_{3}\right) \delta 1.34\left(\mathrm{~s}, 3 \mathrm{H}, \mathrm{CH}_{3}\right), 3.36(\mathrm{~d}$, $1 \mathrm{H}, J=8.0 \mathrm{~Hz}, \mathrm{CH}), 3.56(\mathrm{~d}, 1 \mathrm{H}, J=8.0 \mathrm{~Hz}, \mathrm{CH}), 4.12(\mathrm{~d}, 1 \mathrm{H}, J=$ $4.0 \mathrm{~Hz}, \mathrm{CH}), 4.38$ (d, $1 \mathrm{H}, J=4.0 \mathrm{~Hz}, \mathrm{CH}), 4.56-4.46\left(\mathrm{~m}, 2 \mathrm{H}, \mathrm{CH}_{2}\right)$, $5.18\left(\mathrm{~s}, 2 \mathrm{H}, \mathrm{CH}_{2}\right), 6.84$ (bs, $\left.1 \mathrm{H}, \mathrm{NH}\right), 7.38-7.43\left(\mathrm{~m}, 6 \mathrm{H}, \mathrm{H}_{\mathrm{ar}}\right), 7.63$ $\left(\mathrm{d}, 2 \mathrm{H}, J=4.0 \mathrm{~Hz}, \mathrm{H}_{\mathrm{ar}}\right) ;{ }^{13} \mathrm{C} \mathrm{NMR}\left(100 \mathrm{MHz}, \mathrm{CDCl}_{3}\right) \delta 19.7,61.2$, 67.3, 71.4, 72.5, 125.4, 122.7, 125.5, 125.6, 127.5, 128.8, 129.6, 133.8, 134.4, 141.4, 156.1, 156.7; HRMS calcd for $\mathrm{C}_{21} \mathrm{H}_{21} \mathrm{~N}_{2} \mathrm{O}_{5^{-}}$ $\mathrm{ClF}_{3}[\mathrm{M}+\mathrm{H}]^{+}$473.1084, found 473.1086; HPLC (Daicel Chiralpak AS-H, hexane/isopropanol $=70: 30$, flow rate 1.0 $\mathrm{mL} \min ^{-1}, \lambda=254 \mathrm{~nm}$ ): $t_{\mathrm{R}}=34.57 \mathrm{~min}$ (major), $t_{\mathrm{R}}=56.49 \mathrm{~min}$ (minor).

\section{3-Benzyloxycarbonylamino-4-methyl-4-(4-nitro)benzyloxy- oxazolidin-2-one (5i-Bn)}

Oil, yield 77\%; ${ }^{1} \mathrm{H}$ NMR (400 $\left.\mathrm{MHz}, \mathrm{CDCl}_{3}\right) \delta 1.30\left(\mathrm{~s}, 3 \mathrm{H}, \mathrm{CH}_{3}\right)$, 3.35 (d, $1 \mathrm{H}, J=12.0 \mathrm{~Hz}, \mathrm{CH}), 3.56$ (d, $1 \mathrm{H}, J=12.0 \mathrm{~Hz}, \mathrm{CH}), 4.10$ $(\mathrm{d}, 1 \mathrm{H}, J=4.0 \mathrm{~Hz}, \mathrm{CH}), 4.36(\mathrm{~d}, 1 \mathrm{H}, J=8.0 \mathrm{~Hz}, \mathrm{CH}), 4.54-4.64$ $\left(\mathrm{m}, 2 \mathrm{H}, \mathrm{CH}_{2}\right), 5.13\left(\mathrm{~s}, 2 \mathrm{H}, \mathrm{CH}_{2}\right), 6.82(\mathrm{bs}, 1 \mathrm{H}, \mathrm{NH}), 7.25-7.35(\mathrm{~m}$, $\left.5 \mathrm{H}, \mathrm{H}_{\mathrm{ar}}\right), 7.41\left(\mathrm{~d}, 2 \mathrm{H}, J=8.0 \mathrm{~Hz}, \mathrm{H}_{\mathrm{ar}}\right), 8.15(\mathrm{~d}, 2 \mathrm{H}, J=12.0 \mathrm{~Hz}$, $\left.\mathrm{H}_{\mathrm{ar}}\right) ;{ }^{13} \mathrm{C}$ NMR $\left(100 \mathrm{MHz}, \mathrm{CDCl}_{3}\right) \delta 18.4,19.8,29.7,30.9,61.2$, 68.2, 71.4, 72.1, 73.0, 76.7, 77.1, 77.2, 77.4, 123.8, 127.7, 128.2, 128.6, 128.7, 136.2, 144.8, 147.6, 164.2, 164.6, 207.2; HRMS calcd for $\mathrm{C}_{20} \mathrm{H}_{22} \mathrm{~N}_{3} \mathrm{O}_{7}[\mathrm{M}+\mathrm{H}]^{+}$416.1452, found 416.1435; HPLC (Daicel Chiralpak AD-H, hexane/isopropanol = $90: 10$, flow rate $1.0 \mathrm{~mL} \min ^{-1}, \lambda=254 \mathrm{~nm}$ ): $t_{\mathrm{R}}=72.96 \min$ (major), $t_{\mathrm{R}}=$ $76.57 \mathrm{~min}$ (minor), $55 \%$ ee.

4-Chlorobenzyl((4R)-4-methyl-2-oxo-4-(((tetrahydro-2H-pyran2-yl)oxy)methyl)oxazolidin-3-yl)carbamate (5j-Bn)

Oil, yield 70\%; ${ }^{1} \mathrm{H}$ NMR (400 MHz, $\left.\mathrm{CDCl}_{3}\right): \delta 1.26\left(\mathrm{~s}, 3 \mathrm{H}, \mathrm{CH}_{3}\right.$ ), 1.41-1.56 (m, 4H, $2 \mathrm{CH}_{2}$ ), 1.60-1.74 (m, 4H, $\left.\mathrm{CH}_{2}\right), 3.34-3.84(\mathrm{~m}$, $\left.4 \mathrm{H}, \mathrm{CH}_{2}\right), 4.05-4.11$ (m, 1H, CH), 4.33-4.38 (m, 1H, CH), 4.434.55 (m, 1H, CH), 5.14 (s, 2H, $\mathrm{CH}_{2}$ ), 6.93 (bs, $\left.1 \mathrm{H}, \mathrm{NH}\right), 7.26-7.33$ $\left(\mathrm{m}, 5 \mathrm{H}, \mathrm{H}_{\mathrm{ar}}\right.$ ); HRMS calcd for $\mathrm{C}_{18} \mathrm{H}_{24} \mathrm{~N}_{2} \mathrm{O}_{6} \mathrm{Na}[\mathrm{M}+\mathrm{Na}]^{+} 387.1527$, found 387.1508; HPLC (Daicel Chiralpak $\mathrm{AD}-\mathrm{H}$, hexane/ isopropanol $=90: 10$, flow rate $\left.1.0 \mathrm{~mL} \min ^{-1}, \lambda=254 \mathrm{~nm}\right)$ : $t_{\mathrm{R}}=26.0 \mathrm{~min}$ (major), $t_{\mathrm{R}}=34.5 \mathrm{~min}$ (minor), $60 \%$ ee.

\section{Benzyl(4-ethyl-4-methyl-2-oxooxazolidin-3-yl)carbamate (5l-Bn)}

Oil, yield 78\%; ${ }^{1} \mathrm{H}-\mathrm{NMR}\left(600 \mathrm{MHz}, \mathrm{CDCl}_{3}\right): \delta 0.86-0.96\left(\mathrm{~m}, 3 \mathrm{H}, \mathrm{CH}_{3}\right)$, 1.24-1.33 (s, 3H, $\left.\mathrm{CH}_{3}\right), 1.55-1.70\left(\mathrm{~m}, 2 \mathrm{H}, \mathrm{CH}_{2}\right), 4.06(\mathrm{~d}, J=6.0 \mathrm{~Hz}$, $1 \mathrm{H}, \mathrm{CH}), 4.20(\mathrm{~d}, J=6.0 \mathrm{~Hz}, 1 \mathrm{H}, \mathrm{CH}), 5.19\left(\mathrm{~s}, 2 \mathrm{H}, \mathrm{CH}_{2}\right), 6.53(\mathrm{bs}, 1 \mathrm{H}$, $\mathrm{NH}), 7.26-7.38\left(\mathrm{~m}, 5 \mathrm{H}, \mathrm{H}_{\mathrm{ar}}\right) ;{ }^{13} \mathrm{C} \mathrm{NMR}\left(150 \mathrm{MHz}, \mathrm{CDCl}_{3}\right) \delta$ 7.7, 22.1, 29.8, 53.4, 61.8, 68.2, 72.1, 128.3, 128.5, 128.6, 135.3, 156.0, 156.3; HRMS calcd for $\mathrm{C}_{14} \mathrm{H}_{18} \mathrm{~N}_{2} \mathrm{O}_{4} \mathrm{Na}[\mathrm{M}+\mathrm{Na}]^{+} 301.1159$, found 301.1154; HPLC (Daicel Chiralpak AD-H, hexane/isopropanol $=90: 10$, flow rate $1.0 \mathrm{~mL} \min ^{-1}, \lambda=254 \mathrm{~nm}$ ): $t_{\mathrm{R}}=21.53 \min$ (major), $t_{\mathrm{R}}=$ $23.23 \mathrm{~min}$ (minor), $37 \%$ ee.
Dibenzyl 1-(1-ethoxy-2-methyl-1,3-dioxopropan-2-yl)hydrazine-1,2dicarboxylate (4m-Bn)

Oil, yield 76\%; ${ }^{1} \mathrm{H}-\mathrm{NMR}\left(300 \mathrm{MHz}, \mathrm{CDCl}_{3}\right.$ ): $\delta 1.26(\mathrm{t}, J=6.0 \mathrm{~Hz}$, $\left.3 \mathrm{H}, \mathrm{CH}_{3}\right), 1.56\left(\mathrm{~s}, 3 \mathrm{H}, \mathrm{CH}_{3}\right), 4.18-4.22\left(\mathrm{~m}, 2 \mathrm{H}, \mathrm{CH}_{2}\right), 5.16(\mathrm{~s}, 4 \mathrm{H}$, $2 \mathrm{CH}_{2}$ ), 6.60 (brs, $\left.1 \mathrm{H}, \mathrm{NH}\right), 7.25-7.32\left(\mathrm{~m}, 10 \mathrm{H}, \mathrm{H}_{\mathrm{ar}}\right), 9.60(\mathrm{~s}, 1 \mathrm{H}$, CHO); HRMS calcd for $\mathrm{C}_{22} \mathrm{H}_{25} \mathrm{~N}_{2} \mathrm{O}_{7}[\mathrm{M}+\mathrm{H}]^{+} 429.1653$, found 429.1656; HPLC (Daicel Chiralpak OD-H, hexane/isopropanol = $90: 10$, flow rate $\left.1.0 \mathrm{~mL} \min ^{-1}, \lambda=254 \mathrm{~nm}\right): t_{\mathrm{R}}=20.65 \mathrm{~min}$ (major), $t_{\mathrm{R}}=22.78 \mathrm{~min}$ (minor), $24 \%$ ee.

\section{(R)-3-Amino-4-(hydroxymethyl)-4-methyloxazolidin-2-one (6)}

To a solution of $\mathbf{5 h}$ - $\mathbf{p}$-ClBn $(670 \mathrm{mg}, 1.42 \mathrm{mmol})$ in $8 \mathrm{ml}$ of methanol and acetic acid ( $4 \mathrm{~mL}) .360 \mathrm{mg}$ of $10 \%$ palladium on charcoal was added. The mixture hydrogenated at ambient pressure for $12 \mathrm{~h}$ and filtered. The filtrate was evaporated to dryness under reduced pressure. Column chromatography on silica gel (dichloromethane/methanol, $20: 1$ to $10: 1$ ) delivered $152 \mathrm{mg}$ (1.03 mmol, 73\%) of a colourless solid. Mp $113-115{ }^{\circ} \mathrm{C}$; $[\alpha]_{\mathrm{D}}^{20}-3.86\left(c 0.9, \mathrm{CH}_{3} \mathrm{OH}\right) ;{ }^{1} \mathrm{H} \mathrm{NMR}\left(400 \mathrm{MHz}, \mathrm{CDCl}_{3}\right) \delta 1.20(\mathrm{~s}$, $3 \mathrm{H}, \mathrm{CH}_{3}$ ), 3.31 (dd, $\left.1 \mathrm{H}, J=12.0 \mathrm{~Hz}, 1 \mathrm{~Hz}, \mathrm{CH}\right), 3.55$ (bs, $3 \mathrm{H}, \mathrm{NH}_{2}$ and $\mathrm{OH}), 3.76-3.79(\mathrm{~m}, 1 \mathrm{H}, \mathrm{CH}), 3.96(\mathrm{~d}, 1 \mathrm{H}, J=8.0 \mathrm{~Hz}, \mathrm{CH})$, $4.40(\mathrm{~d}, 1 \mathrm{H}, J=8.0 \mathrm{~Hz}, \mathrm{CH})$; HRMS calcd for $\mathrm{C}_{5} \mathrm{H}_{11} \mathrm{~N}_{2} \mathrm{O}_{3}[\mathrm{M}+\mathrm{H}]^{+}$ 147.0762, found 147.0764 .

\section{(R)-4-(Hydroxymethyl)-4-methyloxazolidin-2-one (7)}

$146 \mathrm{mg}$ ( $1 \mathrm{mmol})$ of $\mathrm{NaNO}_{2}$ was added dropwise to a solution of $45.0 \mathrm{mg}(0.234 \mathrm{mmol})$ of 6 in $18 \mathrm{ml}$ of acetic acid and $6 \mathrm{ml}$ of $1 \mathrm{M} \mathrm{HCl}$. The mixture was refluxed for $1 \mathrm{~h}$. The solvent was evaporated to dryness under reduced pressure. Column chromatography on silica gel (dichloromethane/methanol, $20: 1$ to $10: 1)$ delivered $79 \mathrm{mg}(0.6 \mathrm{mmol}, 60 \%)$ of a white solid. $[\alpha]_{\mathrm{D}}^{20}-8.8\left(c 0.5, \mathrm{CH}_{3} \mathrm{OH}\right) ;{ }^{1} \mathrm{H} \mathrm{NMR}\left(400 \mathrm{MHz}, \mathrm{CDCl}_{3}\right.$ ) $\delta 1.34\left(\mathrm{~s}, 3 \mathrm{H}, \mathrm{CH}_{3}\right), 3.55\left(\mathrm{dd}, 1 \mathrm{H}, J=12.0 \mathrm{~Hz}, 4 \mathrm{~Hz}, \mathrm{CH}_{2}\right), 4.04$ (d, $1 \mathrm{H}, J=8.0 \mathrm{~Hz}, \mathrm{CH}), 4.33$ (d, $1 \mathrm{H}, J=8.0 \mathrm{~Hz}, \mathrm{CH}), 5.59$ (bs, $1 \mathrm{H}, \mathrm{NH}) ;{ }^{13} \mathrm{C} \mathrm{NMR}\left(150 \mathrm{MHz}, \mathrm{CDCl}_{3}\right) \delta 22.6,58.9,67.5,72.8$, 159.4; HRMS calcd for $\mathrm{C}_{5} \mathrm{H}_{10} \mathrm{NO}_{3}[\mathrm{M}+\mathrm{H}]^{+} 132.0654$, found 132.0655 .

\section{4'-(2-Propyloxazol-4-yl)-[1,1'-biphenyl]-4-carbaldehyde (11)}

Catalyst Pd-dimer $(2.5 \mathrm{mg}, 1 \mathrm{~mol} \%$ in respect to 4-bromobenzaldehyde) was added to a suspension of 4-bromobenzaldehyde (101 mg, $0.55 \mathrm{mmol}$ ), $\mathrm{K}_{2} \mathrm{CO}_{3}(207 \mathrm{mg}, 1.5 \mathrm{mmol})$ and 10 (157 mg, $0.5 \mathrm{mmol})$ in toluene : EtOH : $\mathrm{H}_{2} \mathrm{O}=1: 1: 1(\mathrm{v} / \mathrm{v} / \mathrm{v})$. The mixture was refluxed for $4 \mathrm{~h}$. Then the solvent was removed under vacuum. The crude material was extracted with $\mathrm{Et}_{2} \mathrm{O}$ and washed with brine. The organic phase was dried $\left(\mathrm{Na}_{2} \mathrm{SO}_{4}\right)$ and the solvent was evaporated under reduced pressure. The crude product was chromatographed (silica gel, light petroleum ether/ethyl acetate $=20: 1)$ to afford the aldehyde $(116 \mathrm{mg}, 80 \%)$ as a white solid. Mp $100{ }^{\circ} \mathrm{C} ;{ }^{1} \mathrm{H}$ NMR (400 MHz, $\left.\mathrm{CDCl}_{3}\right) \delta 1.04(\mathrm{t}, 3 \mathrm{H}, J=8.0 \mathrm{~Hz}$, $\left.\mathrm{CH}_{3}\right)$, 1.84-1.89 (m, 2H, $\left.\mathrm{CH}_{2}\right), 2.83\left(\mathrm{~m}, 2 \mathrm{H}, \mathrm{CH}_{2}\right), 7.69(\mathrm{~m}, 2 \mathrm{H}$, $\mathrm{H}_{\mathrm{ar}}$ ), 7.78-7.85 (m, 4H, $\left.\mathrm{H}_{\mathrm{ar}}\right), 7.89\left(\mathrm{~s}, 1 \mathrm{H}, \mathrm{H}_{\mathrm{ar}}\right), 7.95-8.01(\mathrm{~m}, 4 \mathrm{H}$, $\mathrm{H}_{\mathrm{ar}}$ ), 10.06 (s, 1H, CHO); HRMS calcd for $\mathrm{C}_{19} \mathrm{H}_{18} \mathrm{NO}_{2}[\mathrm{M}+\mathrm{H}]^{+}$ 292.1332, found 292.1335. 
$(R, E)$-4-Methyl-4-(2-(4'-(2-propyloxazol-4-yl)-[1,1'-biphenyl]4-yl)vinyl)oxazolidin-2-one (12)

To a suspension of the phosphonium salt $(235 \mathrm{mg}, 0.48 \mathrm{mmol})$ in THF was added $n$-butyllithium (2.5 $\mathrm{M}$ in hexane, $0.37 \mathrm{~mL}$, $0.937 \mathrm{mmol}$ ) at $-78{ }^{\circ} \mathrm{C}$ and then the solution was stirred for $30 \mathrm{~min}$ at the same temperature. After the addition of benzaldehyde ( $70 \mathrm{mg}, 0.24 \mathrm{mmol}$ ) at $-78{ }^{\circ} \mathrm{C}$, the reaction mixture was warmed to ambient temperature and stirred for $3 \mathrm{~h}$. After quenching with saturated aq. $\mathrm{NH}_{4} \mathrm{Cl}$, the resulting biphasic mixture was extracted with AcOEt. The combined organic layer was washed with water and brine, dried over $\mathrm{Na}_{2} \mathrm{SO}_{4}$, filtered, and evaporated. Purification by silica gel column chromatography (hexane : AcOEt $=4: 1$ to $1: 1)$ provided $12(131 \mathrm{mg}$, $73 \%)$ as a white solid. $\mathrm{Mp} 235{ }^{\circ} \mathrm{C} ;[\alpha]_{\mathrm{D}}^{20}-17.8\left(c 0.1, \mathrm{CH}_{3} \mathrm{OH}\right) ;{ }^{1} \mathrm{H}$ NMR (400 MHz, $\mathrm{CDCl}_{3}$ ) $\delta 1.03\left(\mathrm{t}, 3 \mathrm{H}, J=8.0 \mathrm{~Hz}, \mathrm{CH}_{3}\right.$ ), 1.81-1.90 $\left(\mathrm{m}, 2 \mathrm{H}, \mathrm{CH}_{2}\right), 2.82\left(\mathrm{t}, 2 \mathrm{H}, J=8.0 \mathrm{~Hz}, \mathrm{CH}_{2}\right), 4.17-4.21(\mathrm{~m}, 1 \mathrm{H}$, $\mathrm{CH}), 4.58-4.65\left(\mathrm{~m}, 2 \mathrm{H}, \mathrm{CH}_{2}\right), 5.12(\mathrm{~s}, 1 \mathrm{H}, \mathrm{NH}), 6.16-6.22(\mathrm{~m}, 1 \mathrm{H}$, $\mathrm{CH}), 6.66(\mathrm{~d}, 1 \mathrm{H}, J=16.0 \mathrm{~Hz}, \mathrm{CH}), 7.46\left(\mathrm{~d}, 2 \mathrm{H}, J=8.0 \mathrm{~Hz}, \mathrm{H}_{\mathrm{ar}}\right.$ ), 7.59-7.65 (m, $\left.4 \mathrm{H}, \mathrm{H}_{\mathrm{ar}}\right), 7.80\left(\mathrm{~d}, 2 \mathrm{H}, J=8.0 \mathrm{~Hz}, \mathrm{H}_{\mathrm{ar}}\right), 7.87(\mathrm{~s}, 1 \mathrm{H}$, $\left.\mathrm{H}_{\mathrm{ar}}\right) ;{ }^{13} \mathrm{C} \mathrm{NMR}\left(100 \mathrm{MHz}, \mathrm{CDCl}_{3}\right) \delta 13.7,20.7,30.2,56.2,70.2$, $125.9,126.3,127.2,127.3,130.6$, 133.2, 133.7, 134.3, 139.6, 140.1, 140.9, 158.9, 165.5; HRMS calcd for $\mathrm{C}_{24} \mathrm{H}_{25} \mathrm{~N}_{2} \mathrm{O}_{3}[\mathrm{M}+\mathrm{H}]^{+}$ 389.1860 , found 389.1882 .

\section{(R)-4-Methyl-4-(2-(4'-(2-propyloxazol-4-yl)-[1,1'-biphenyl]-4-yl)ethyl)} oxazolidin-2-one (13)

To a solution of 12 (120 mg, $0.32 \mathrm{mmol})$ in methanol was added $10 \% \mathrm{Pd} / \mathrm{C}(30 \mathrm{mg})$, and then the suspension was stirred for $2 \mathrm{~h}$ under a hydrogen atmosphere at ambient temperature. The reaction mixture was filtered and evaporated in vacuo, providing the product $13(112 \mathrm{mg}, 92 \%)$ as a white solid. Mp $185{ }^{\circ} \mathrm{C}$; $[\alpha]_{\mathrm{D}}^{20} 14.4\left(c 0.5, \mathrm{CHCl}_{3}\right) ;{ }^{1} \mathrm{H} \mathrm{NMR}\left(400 \mathrm{MHz}, \mathrm{CDCl}_{3}\right) \delta 1.04(\mathrm{t}, 3 \mathrm{H}$, $\left.J=8.0 \mathrm{~Hz}, \mathrm{CH}_{3}\right), 1.44\left(\mathrm{~s}, 3 \mathrm{H}, \mathrm{CH}_{3}\right), 1.84-1.89\left(\mathrm{~m}, 2 \mathrm{H}, \mathrm{CH}_{2}\right), 1.93-$ $1.97\left(\mathrm{~m}, 2 \mathrm{H}, \mathrm{CH}_{2}\right), 2.70-2.74\left(\mathrm{~m}, 2 \mathrm{H}, \mathrm{CH}_{2}\right), 2.75-2.86(\mathrm{~m}, 2 \mathrm{H}$, $\mathrm{CH}_{2}$ ), 4.10 (d, $\left.1 \mathrm{H}, J=8.0 \mathrm{~Hz}, \mathrm{CH}\right), 4.22(\mathrm{~d}, 1 \mathrm{H}, J=8.0 \mathrm{~Hz}, \mathrm{CH})$, 5.42 (bs, 1H, NH), 7.25 (d, 1H, $J=8.0 \mathrm{~Hz}, \mathrm{CH}), 7.55$ (d, 1H, $J=$ $8.0 \mathrm{~Hz}, \mathrm{CH}), 7.61\left(\mathrm{~d}, 2 \mathrm{H}, J=8.0 \mathrm{~Hz}, \mathrm{H}_{\mathrm{ar}}\right), 7.79(\mathrm{~m}, 2 \mathrm{H}, J=8.0 \mathrm{~Hz}$, $\left.\mathrm{H}_{\mathrm{ar}}\right), 7.86\left(\mathrm{~s}, 1 \mathrm{H}, \mathrm{H}_{\mathrm{ar}}\right) ;{ }^{13} \mathrm{C} \mathrm{NMR}\left(100 \mathrm{MHz}, \mathrm{CDCl}_{3}\right) \delta 13.7,20.6$, 26.0, 30.0, 30.1, 42.2 , 57.6, 75.6, 125.9, 127.2, 127.3, 128.7, 129.8, 133.2, 138.8, 139.8, 139.9, 140.3, 158.7, 165.6; HRMS calcd for $\mathrm{C}_{24} \mathrm{H}_{27} \mathrm{~N}_{2} \mathrm{O}_{3}[\mathrm{M}+\mathrm{H}]^{+}$391.2016, found 391.2011.

(R)-2-Amino-2-methyl-4-(4' -(2-propyloxazol-4-yl)-[1,1'-biphenyl]4-yl)butan-1-ol hydrochloride (14)

Compound 13 (100 mg, $0.26 \mathrm{mmol}$ ) was diluted with methanol $: \mathrm{H}_{2} \mathrm{O}=10: 1(\mathrm{v} / \mathrm{v})$, then potassium hydroxide (146 mg, $2.6 \mathrm{mmol}$ ) was added, which was refluxed for $18 \mathrm{~h}$. After cooling to room temperature, water was added to the reaction mixture and extracted with $\mathrm{CH}_{2} \mathrm{Cl}_{2}$. The combined organic layer was washed with water and brine, dried over $\mathrm{Na}_{2} \mathrm{SO}_{4}$, filtered, and evaporated. The crude product was chromatographed (silica gel, dichloromethane/methanol = $10: 1$ ), then added $1 \mathrm{M} \mathrm{HCl}$ in $\mathrm{Et}_{2} \mathrm{O}(2 \mathrm{~mL})$ to afford the product 14 $(82 \mathrm{mg}, 80 \%)$ as a white solid. $\mathrm{Mp} 214{ }^{\circ} \mathrm{C} ;[\alpha]_{\mathrm{D}}^{20}-1.61(c 0.2$, $\left.\mathrm{CH}_{3} \mathrm{OH}\right) ;{ }^{1} \mathrm{H}$ NMR $\left(400 \mathrm{MHz}, \mathrm{CDCl}_{3}\right) \delta 1.01(\mathrm{t}, 3 \mathrm{H}, J=8.0 \mathrm{~Hz}$,
$\left.\mathrm{CH}_{3}\right), 1.34\left(\mathrm{~s}, 3 \mathrm{H}, \mathrm{CH}_{3}\right), 1.81-1.92\left(\mathrm{~m}, 2 \mathrm{H}, \mathrm{CH}_{2}\right), 2.68-2.72(\mathrm{~m}$, $\left.2 \mathrm{H}, \mathrm{CH}_{2}\right), 2.83-2.86\left(\mathrm{~m}, 2 \mathrm{H}, \mathrm{CH}_{2}\right), 3.52-3.57\left(\mathrm{~m}, 2 \mathrm{H}, \mathrm{CH}_{2}\right), 3.62-$ $3.65\left(\mathrm{~m}, 2 \mathrm{H}, \mathrm{CH}_{2}\right), 7.31\left(\mathrm{~d}, 2 \mathrm{H}, J=8.0 \mathrm{~Hz}, \mathrm{H}_{\mathrm{ar}}\right), 7.58(\mathrm{~d}, 2 \mathrm{H}, J=$ $8.0 \mathrm{~Hz}, \mathrm{H}_{\mathrm{ar}}$ ), 7.65 (d, $\left.2 \mathrm{H}, J=8.0 \mathrm{~Hz}, \mathrm{H}_{\mathrm{ar}}\right), 7.77$ (d, $2 \mathrm{H}, J=8.0 \mathrm{~Hz}$, $\mathrm{H}_{\mathrm{ar}}$ ), $8.23\left(\mathrm{~s}, 1 \mathrm{H}, \mathrm{H}_{\mathrm{ar}}\right) ;{ }^{13} \mathrm{C} \mathrm{NMR}\left(100 \mathrm{MHz}, \mathrm{CDCl}_{3}\right) \delta 14.0,20.4$, 21.6, 30.2, 30.8, 38.7, 58.8, 66.3, 127.2, 128.2, 128.3, 130.0, 136.0, 139.9, 140.7, 141.9, 142.1, 167.8; HRMS calcd for $\mathrm{C}_{23} \mathrm{H}_{29} \mathrm{~N}_{2} \mathrm{O}_{2}[\mathrm{M}$ $+\mathrm{H}]^{+}$365.2224, found 365.2212.

\section{Conflicts of interest}

There are no conflicts to declare.

\section{Acknowledgements}

This work was financially supported by the Drug Innovation Major Project (No. 2018ZX09711001-005-012), National Key R\&D Program of China (No. 2018YFC1706403) and CAMS Innovation Fund for Medical Sciences (No. 2016-I2M-2-002).

\section{Notes and references}

1 E. Katz, H. Schmitt, M. Aydin, W. A. König and G. Jung, Liebigs Ann. Chem., 1985, 365.

2 C. Auvin-Guette, S. Rebuffat, I. Vuidepot, M. Massias and B. Bodo, J. Chem. Soc., Perkin Trans. 1, 1993, 249.

3 I. Augeven-Bour, S. Rebuffat, C. Auvin, C. Goulard, Y. Prigent and B. Bodo, J. Chem. Soc., Perkin Trans. 1, 1997, 1587.

4 M. Ahrend, Angew. Chem., 1999, 111, 3047; Angew. Chem., Int. Ed., 1999, 38, 2873.

5 K. L. Reddy and K. B. Sharpless, J. Am. Chem. Soc., 1998, 120, 1207.

6 H. F. Wang, G. H. Ma, S. B. Yang, R. G. Han and P. F. Xu, Tetrahedron: Asymmetry, 2008, 19, 1630.

7 G. P. Miley, J. C. Rote, R. B. Silverman, N. L. Kelleher and R. J. Thomson, Org. Lett., 2018, 20, 2369.

8 T. Tsuji, K. Suzuki, T. Nakamura and T. Nishi, Tetrahedron, 2014, 70, 5234.

9 M. A. Jones, A. D. Hislop and J. S. Snaith, Org. Biomol. Chem., 2006, 4, 3769.

10 D. Seebach, J. D. Aebi, M. Gander-Coquoz and R. Naef, Helv. Chim. Acta, 1987, 70, 1194.

11 M. Di Giacomo, V. Vinci, M. Serra and L. Colombo, Tetrahedron: Asymmetry, 2008, 19, 247.

12 P. Vachal and E. N. Jacobsen, Org. Lett., 2000, $2,867$.

13 K. Maruoka and T. Ooi, Chem. Rev., 2003, 103, 3013.

14 H. Vogt, S. Vanderheiden and S. Bräse, Chem. Commun., 2003, 19, 2448.

15 N. Kumaragurubaran, K. Juhl, W. Zhuang, A. Bøgevig and K. A. Jørgensen, J. Am. Chem. Soc., 2002, 124, 6254.

16 C. E. Hartmann, T. Baumann, M. Bächle and S. Bräse, Tetrahedron: Asymmetry, 2010, 21, 1341.

17 J. Ferreira, S. C. Rees-Jones, V. Ramaotsoa and R. Hunter, Org. Biomol. Chem., 2016, 14, 1545.

18 J. Y. Fu, Q. C. Yang, Q. L. Wang, J. N. Ming, F. Y. Wang, X. Y. Xu and L. X. Wang, J. Org. Chem., 2011, 76, 4661.

19 N. S. Chowdari and C. F. Barbas, Org. Lett., 2005, 7, 867. 
20 T. Tsuji, K. Suzuki, T. Nakamura and T. Nishi, Tetrahedron, 2014, 70, 5234.

21 H. Deng, S. G. Bernier, E. Doyle, J. Lorusso, B. A. Morgan, W. F. Westlin and G. Evindar, ACS Med. Chem. Lett., 2013, 4, 942.

22 T. Nishi, S. Miyazaki, T. Takemoto, K. Suzuki, Y. Iio, K. Nakajima, T. Ohnuki, Y. Kawase, F. Nara, S. Inaba,
T. Izumi, H. Yuita, K. Ohshima, H. Doi, R. Inoue, W. Tomisato, T. Kagari and T. Shimozato, ACS Med. Chem. Lett., 2011, 2, 368.

23 J. Jin, J. P. Hu, W. Q. Zhou, X. J. Wang, Q. Xiao, N. N. Xue, D. L. Yin and X. G. Chen, Biochem. Pharmacol., 2014, 90, 50. 24 S. Chen, et al., under review. 